\title{
Spectral asymptotics via the semiclassical Birkhoff normal form
}

\author{
Laurent Charles* $\quad$ VŨ NGỌ San ${ }^{\dagger}$
}

July 10, 2018

Keywords : Birkhoff normal form, resonances, pseudo-differential operators, spectral asymptotics, symplectic reduction, Toeplitz operators, eigenvalue cluster.

MS Classification : 58J40, 58J50, 58K50, 47B35, 53D20, 81S10.

\begin{abstract}
This article gives a simple treatment of the quantum Birkhoff normal form for semiclassical pseudo-differential operators with smooth coefficients. The normal form is applied to describe the discrete spectrum in a generalised non-degenerate potential well, yielding uniform estimates in the energy $E$. This permits a detailed study of the spectrum in various asymptotic regions of the parameters $(E, \hbar)$, and gives improvements and new proofs for many of the results in the field. In the completely resonant case we show that the pseudo-differential operator can be reduced to a Toeplitz operator on a reduced symplectic orbifold. Using this quantum reduction, new spectral asymptotics concerning the fine structure of eigenvalue clusters are proved. In the case of polynomial differential operators, a combinatorial trace formula is obtained.
\end{abstract}

\section{Introduction}

The Birkhoff normal form, in classical mechanics, is a well known refinement of the averaging method : under a suitable canonical transformation, a perturbation of a harmonic oscillator $H_{2}$ can be replaced by its average along the classical Hamiltonian flow generated by $\mathrm{H}_{2}$. With the averaging method, this remains valid as long as one restricts the dynamics to times bounded by $\mathscr{O}(1 / \epsilon)$, where $\epsilon$ is the size of the perturbation. Using the Birkhoff normal form, this time can be extended to $\mathscr{O}\left(1 / \epsilon^{N}\right)$ for arbitrary $N$, provided one takes into account higher order terms which are also averaged, but in a

\footnotetext{
*Institut de Mathématiques de Jussieu (UMR 7586), Université Pierre et Marie Curie - Paris 6, Paris, F-75005 France.

${ }^{\dagger}$ Institut Fourier (UMR 5582), Université Joseph Fourier - Grenoble 1, BP 74, 38402Saint Martin d'Hères Cedex, France.
} 
more intricate sense. Note that, in this work, we do not try to impose special restrictions to the original Hamiltonian that would imply some better convergence properties (Gevrey convergence, or even analyticity). Instead, we take any smooth function and perform the Birkhoff normal form in a neighbourhood of a non-degenerate minimum.

In quantum mechanics, it is known since at least 1975 that an analogue of the Birkhoff normal form can be applied in a very successful way. At the formal level, this is attested by physicists like [13, 1. Adding on top of this the experience of excellent numerical computations, it has become an important tool for molecular physics (see [28] and more recently [20, 24]).

On the mathematics side, the Birkhoff normal form for pseudo-differential operators near a non-degenerate minimum of the symbol has been used by several authors already. In particular the article [27] by Sjöstrand is very interesting with this respect, but only deals with the non-resonant normal form, that it, when the harmonic oscillator is of the form

$$
H_{2}(x, \xi)=\sum_{j=1}^{n} \nu_{j}\left(x_{j}^{2}+\xi_{j}^{2}\right) / 2,
$$

where the coefficients $\nu_{j}$ are linearly independent over the rationals. The result is that, when the energy $E$ is of order $\hbar^{\gamma}$ with $\gamma \in(0,1)$ then, for $\hbar$ small enough, the quantum system has the same spectrum as a completely integrable Hamiltonian.

The initial goal of our work here is to extend this to the resonant case. But since our methods also give new proofs for Sjöstrand's result and some improvements, and moreover unify them with the analysis of low-lying eigenvalues initially discovered by Simon [26] and Helffer-Sjöstrand [19], it might be of interest to present it here in the general case. Moreover, we believe that several intermediate statements are of independent value, and involve for the main part only standard results of semiclassical analysis (symbolic and functional calculus for pseudo-differential operators). In particular we are able to compare the initial pseudo-differential operator to a differential operator with polynomial coefficients, which is very important for many practical purposes, including numerical computations. On the other hand, the treatment of the resonant case is very hard to perform within the standard pseudo-differential calculus because of unavoidable singularities due to the fact that, when the coefficients $\nu_{j}$ are integers, the $S^{1}$-action generated by the time- $2 \pi$ flow of $H_{2}$ is in general non free : periodic orbits with smaller periods appear. This explains why so little results were available in that case. Our strategy here is to abandon pseudo-differential operators for Toeplitz operators, in the spirit of Boutet de Monvel and Guillemin [6]. The appropriate theory that can deal with orbifold singularities was developed in [7] and [8]. 
Let us briefly describe our spectral result in this case. Assume $P=$ $-\frac{h^{2}}{2} \Delta+V(x)$ is a Schrödinger operator with a smooth potential $V$ on $X=\mathbb{R}^{n}$ or on an $n$-dimensional compact manifold $X$ equipped with a smooth density. (More generally, $P$ could be any pseudo-differential operator in some standard class, which is actually our assumption in this article.) Assume $V \in C^{\infty}(X)$ has a global minimum at a point 0 which we shall call here the origin, and suppose this minimum is non-degenerate. By a linear, unitary change of variable in local coordinates near 0 , one can always assume that $V^{\prime \prime}(0)$ is diagonal; let $\left(\nu_{1}^{2}, \ldots, \nu_{n}^{2}\right)$ be its eigenvalues, with $\nu_{j}>0$. The rescaling $x_{j} \mapsto \sqrt{\nu_{j}} x_{j}$ transforms $P$ into a perturbation of the harmonic oscillator $\hat{H}_{2}$ :

$$
P=\hat{H}_{2}+W(x), \quad \text { with } \hat{H}_{2}=\sum_{i=1}^{n} \frac{\nu_{j}}{2}\left(-\hbar^{2} \frac{\partial^{2}}{\partial x_{j}^{2}}+x_{j}^{2}\right),
$$

where $W(x)$ is a smooth potential of order $\mathscr{O}\left(|x|^{3}\right)$ at the origin.

Now assume that the coefficients $\nu_{j}$ are completely resonant: there exist a real number $\nu_{c}>0$ and coprime positive integers $\mathfrak{p}_{1}, \ldots, \mathfrak{p}_{n}$ such that $\nu_{j}=\nu_{c} \mathfrak{p}_{j}$. Then the spectrum of $\hat{H}_{2}$ consists of the arithmetic progression $E_{N}=\hbar \nu_{c}\left(\frac{|\nu|}{2}+N\right)$ for $N \in \mathbb{N}$, with multiplicity of order $N^{n-1}$ as $N \rightarrow \infty$. It is then expected that, for small energies, the spectrum of $P$ is a perturbation of the spectrum of $\hat{H}_{2}$, splitting each eigenvalue $E_{N}$ into a band, or cluster. We prove this in a precise way. Actually, we describe in theorem 5.3 the size and the internal structure of each cluster, as follows. Let $H_{2}$ be the corresponding classical harmonic oscillator, as in (1). It has a $2 \pi$ periodic Hamiltonian flow $\varphi_{t}$. Let $k=k(x, \xi)$ be the average of $W$ along this flow.

$$
k(x, \xi)=\frac{1}{2 \pi} \int_{0}^{2 \pi} W \circ \varpi\left(\varphi_{t}(x, \xi)\right)
$$

where $\varpi: T^{*} X \rightarrow X$ is the cotangent projection. Let $S_{N} \subset T^{*} X$ be the sphere:

$$
S_{N}=\left\{(x, \xi) \in T^{*} X, \quad H_{2}(x, \xi)=E_{N}\right\} .
$$

Theorem [theorem 5.3]

1. There exists $\hbar_{0}>0$ and $C>0$ such that for every $\hbar \in\left(0, \hbar_{0}\right]$

$$
\operatorname{Sp}(P) \cap\left(-\infty, C \hbar^{\frac{2}{3}}\right) \subset \bigcup_{E_{N} \in \operatorname{Sp}\left(\hat{H}_{2}\right)}\left[E_{N}-\frac{\nu_{c} \hbar}{3}, E_{N}+\frac{\nu_{c} \hbar}{3}\right] .
$$

2. When $E_{N} \leqslant C \hbar^{\frac{2}{3}}$, let $m\left(E_{N}, \hbar\right)=\# \operatorname{Sp}(P) \cap\left[E_{N}-\frac{\nu_{c} \hbar}{3}, E_{N}+\frac{\nu_{c} \hbar}{3}\right]$. Then $m\left(E_{N}, \hbar\right)$ is precisely the dimension of $\operatorname{ker}\left(\hat{H}_{2}-E_{N}\right)$. 
3. Let $E_{N}+\lambda_{1}\left(E_{N}, \hbar\right), \ldots, E_{N}+\lambda_{m\left(E_{N}, \hbar\right)}\left(E_{N}, \hbar\right)$ be the eigenvalues of $P$ in this $N$-eth band. Then, uniformly for $\hbar<\hbar_{0}$ and $N$ such that $E_{N} \leqslant C \hbar^{\frac{2}{3}}$,

$$
\begin{aligned}
\lambda_{1}\left(E_{N}, \hbar\right) & =\inf _{(x, \xi) \in S_{N}}|k(x, \xi)|+\left(E_{N}\right)^{\frac{3}{2}} \mathscr{O}\left(N^{-1}\right), \\
\lambda_{m\left(E_{N}, \hbar\right)}\left(E_{N}, \hbar\right) & =\sup _{(x, \xi) \in S_{N}}|k(x, \xi)|+\left(E_{N}\right)^{\frac{3}{2}} \mathscr{O}\left(N^{-1}\right)
\end{aligned}
$$

and for any function $g \in C^{\infty}(\mathbb{R})$,

$$
\sum_{i=1}^{m\left(E_{N}, \hbar\right)} g\left(\frac{\lambda_{i}\left(E_{N}, \hbar\right)}{\left(E_{N}\right)^{\frac{3}{2}}}\right)=\left(\frac{1}{2 \pi \hbar}\right)^{n-1} \int_{S_{N}} g\left(\frac{k(x, \xi)}{\left(E_{N}\right)^{\frac{3}{2}}}\right) \mu_{E_{N}}(x, \xi)+\mathscr{O}\left(N^{2-n}\right)
$$

where $\mu_{E_{N}}$ is the Liouville measure of $S_{N}$

Thus we see that the average perturbation $k$ behaves as a principal symbol for the spectral analysis restricted to each cluster. Several improvements of this statement are proved in the article. First, $k$ can actually be replaced by the homogeneous term of degree 3 in its Taylor expansion. Secondly, the exponent $2 / 3$ in the term $C \hbar^{2 / 3}$ (and in $\left(E_{N}\right)^{3 / 2}$ where its inverse appears) is due to the fact that in general resonances of order 3 may happen in $\mathrm{H}_{2}$ : relations of the form $\mathfrak{p}_{j}=2 \mathfrak{p}_{i}$ or $\mathfrak{p}_{i}=\mathfrak{p}_{j}+\mathfrak{p}_{k}$. If one rules these out, then the exponent $2 / 3$ can be replaced by $1 / 2$, but in general with a modified $k$ (if the homogeneous term of degree 3 in the Taylor expansion of the potential vanishes, then $k$ keeps the same definition. Otherwise the formula is more involved). Finally, the last expansion in the theorem is actually the leading order of a full asymptotic expansion in $\hbar / E$. In particular, in sub-principal terms, one can exhibit oscillatory contributions of type $\zeta^{N}$ where $\zeta$ is some (finite order) complex root of 1 .

The estimate of the spectral density in the particular case $\mathfrak{p}_{1}=\ldots=$ $\mathfrak{p}_{n}=1$ was first obtained in the thesis of the second author 29] through a reduction to Toeplitz operators. Independently Bambusi and Tagliaferro conjectured and partially proved the estimates of the smallest and largest eigenvalues in each band. Then Bambusi and the first author worked on a proof using the quantum Birkhoff normal form of [3] and Toeplitz operators. The result was announced in [2].

On technical side, it might be worth mentioning here that we do not use any exotic pseudo-differential calculus in order to deal with formal Taylor series. Instead of this, we rely extensively on various scaling properties of the harmonic oscillator $H_{2}$, which seems particularly fit for this purpose. This allows us to play all along with $(E, \hbar)$ as two (almost) independent small parameters. The results of Sjöstrand are thus recovered in the regime $E=\hbar^{\gamma}$. Then, when we study the resonant case, these scaling properties of 
$H_{2}$ become even more crucial, because the effective semiclassical parameter becomes $h=\hbar / E$ (sections 国 and 司).

To conclude this introduction, let us mention that quantum Birkhoff normal forms have also become a very important tool in inverse spectral problems. Formally, the Birkhoff normal form is a (semi)classical invariant from which, generally under analyticity assumptions, one can hope to recover the full classical dynamics (see for instance [16] and [30]). This aspect is not discussed here.

Structure of the article. - The Birkhoff normal form is based on a simple formal construction, which can be explained directly in a quantum setting; that's what we recall in section 2 (theorem 2.1). Most of the material in this section is not new; however it is crucial here, and the notation introduced there is used throughout the article. As we next show in section 3, the relevance of the formal result to semiclassical operators is due to a general theorem allowing to compare the spectrum of pseudo-differential operators in the so-called semi-excited regime on the basis of the Taylor expansions of the symbols (theorem 3.1). Adding standard arguments of spectral theory we obtain a general statement of the quantum Birkhoff normal form (theorem 3.11). Up to an error of size $\mathscr{O}\left(E^{\infty}\right)+\mathscr{O}\left(\hbar^{\infty}\right)$, it reduces the spectral problem to the analysis of a pseudo-differential operator $K \mathrm{com}$ muting with a quantum harmonic oscillator $\hat{H}_{2}$. In section 1 we describe the joint spectrum of $P$ and its Birkhoff normalisation $K$, proving an important estimate relating the formal order of $K$ with its operator norm, when restricted to eigenspaces of $\hat{H}_{2}$ (lemma 4.2). Several applications are given : Weyl asymptotics, expansions of the low-lying eigenvalues, and the use of polynomial differential operators (thus giving a rigorous justification of the spectroscopy computations of [28, 20]). Finally the last section 5 is devoted to the resonant case, when $\nu_{j}$ are integers, up to a common multiple. Then the classical flow of $\mathrm{H}_{2}$ is periodic, and it is known that one expects the spectrum to exhibit clustering. We describe these clusters of eigenvalues. Technically and conceptually, the main result is that the restriction of $K$ to eigenspaces of $\hat{H}_{2}$ can be identified to a Toeplitz operator on the corresponding reduced symplectic orbifold (theorem 5.1). This allows us to introduce $\hbar / E$ as a second semiclassical parameter and yields spectral asymptotics for these clusters in terms of the principal symbol of $K$ (theorem 5.3). A more precise trace formula involving sub-orbifolds and hence oscillatory terms is given in 5.5. We end the article with an amusing combinatorial formula expressing a certain sum over integral points of a rational polytope, which comes as a direct consequence of our results (theorem 5.7). 
Acknowledgements - Laurent Charles thanks Dario Bambusi for collaborating on the subject.

\section{The formal Birkhoff normal form}

The Weyl quantisation on $\mathbb{R}^{2 n}=T^{*} \mathbb{R}^{n}$ is based on a particular grading for formal symbols in $x, \xi, \hbar$, where the degree in the semiclassical parameter counts twice the degree of each other variable $x_{i}$ or $\xi_{i}$. This grading is particularly adapted to the harmonic oscillator and hence to the quantum Birkhoff normal form. It also appears naturally in the context of deformation quantisation 14. We mainly follow here the presentation of 29], but other authors have used this approach.

Thus we work with the space

$$
\mathscr{E}=\mathbb{C} \llbracket x_{1}, \ldots, x_{n}, \xi_{1}, \ldots, \xi_{n}, \hbar \rrbracket
$$

and we define the weight of the monomial $x^{\alpha} \xi^{\beta} \hbar^{\ell}$ to be $|\alpha|+|\beta|+2 \ell$. The finite dimensional vector space spanned by monomials of weight $N$ shall be denoted by $\mathscr{D}_{N}$. Let $\mathscr{O}_{N}$ be the subspace consisting of formal series whose coefficients of weight $<N$ vanish. $\left(\mathscr{O}_{N}\right)_{N \in \mathbb{N}}$ is a filtration

$$
\mathscr{E}=\mathscr{O}_{0} \supset \mathscr{O}_{1} \supset \cdots, \quad \bigcap_{N} \mathscr{O}_{N}=\{0\},
$$

and shall be used for all convergences in this section.

The bracket associated to the Weyl product on $\mathscr{E}$ defines a Poisson algebra structure on $\mathscr{E}$ : it is the unique bilinear bracket for which $\hbar$ is central, which satisfies the Jacobi identity, the Leibniz identity (with the Weyl product), and which is commutative on all generators amongst $x_{1}, \ldots, x_{n}$, $\xi_{1}, \ldots, \xi_{n}$ and $\hbar$, except for the relations

$$
\forall j=1, \ldots, n, \quad\left[\xi_{j}, x_{j}\right]=\frac{\hbar}{i} .
$$

Notice that this structure is invariant by linear canonical changes of coordinates. There is a simple formula (Moyal's formula) for the brackets of two elements of $\mathscr{E}$, but we shall not need it in this article. However in the following sections we will use the fact that if $\hat{H}$ and $\hat{P}$ are Weyl-quantisations of symbols $H$ and $P$ with formal Taylor series at the origin $[H]$ and $[P]$ in $\mathscr{E}$, then the Taylor series of the symbol of the operator commutator $[\hat{H}, \hat{P}]$ is precisely the Weyl bracket $[[H],[P]]$.

The filtration of $\mathscr{E}$ has a nice behaviour with respect to the Weyl bracket. If $N_{1}+N_{2} \geqslant 2$ then

$$
\hbar^{-1}\left[\mathscr{O}_{N_{1}}, \mathscr{O}_{N_{2}}\right] \subset \mathscr{O}_{N_{1}+N_{2}-2}
$$


If $A \in \mathscr{E}$ the adjoint operator $P \mapsto[A, P]$ will be denoted by $\operatorname{ad}_{A}$. We shall be interested in the adjoint action of elements of $\mathscr{D}_{2}$. Such elements are of the form $\hbar H_{0}+H_{2}$, where $H_{0} \in \mathbb{C}$ and $H_{2}$ is a quadratic form in $(x, \xi)$. Since $\hbar$ is central, we may restrict here to quadratic forms only. They will be called elliptic when the quadratic form is positive. Because of this grading we see that when $H_{2} \in \mathscr{D}_{2}$, then $\hbar^{-1} \operatorname{ad}_{H_{2}}$ acts as an endomorphism of each $\mathscr{D}_{N}$. A fundamental property of the Weyl bracket is that $\frac{i}{\hbar} \operatorname{ad}_{H_{2}} P$ is exactly the classical Poisson bracket $\left\{H_{2}, P\right\}$.

We will say that $H_{2} \in \mathscr{D}_{2}$ is admissible when $\mathscr{D}_{N}=\operatorname{ker}\left(\operatorname{ad}_{H_{2}}\right)+\operatorname{im}\left(\operatorname{ad}_{H_{2}}\right)$. A typical example is the harmonic oscillator (see lemma 2.5 below):

$$
H_{2}=\nu_{1}\left(x_{1}^{2}+\xi_{1}^{2}\right) / 2+\cdots+\nu_{n}\left(x_{n}^{2}+\xi_{n}^{2}\right) / 2 .
$$

One can show that all elliptic $H_{2}$ can be written as harmonic oscillators in some canonical coordinates and hence are admissible as well. Indeed, eigenvalues of Hamiltonian matrices come by pairs $\left(\nu_{i},-\nu_{i}\right)$ : this implies that an elliptic $H_{2}$ must have the form of a harmonic oscillator plus some nilpotent terms. But no such nilpotent term is allowed to show up because the flow of $H_{2}$ is contained in the hypersurface $\left\{H_{2}=\right.$ const $\}$, which is compact.

The formal quantum Birkhoff normal form can be expressed as follows.

Theorem 2.1 Let $H_{2} \in \mathscr{D}_{2}$ be admissible and $L \in \mathscr{O}_{3}$. Then there exists $A \in \mathscr{O}_{3}$ and $K \in \mathscr{O}_{3}$ such that

$$
\text { - } e^{i \hbar^{-1} \operatorname{ad}_{A}}\left(H_{2}+L\right)=H_{2}+K \text {; }
$$

- $\left[K, H_{2}\right]=0$.

Moreover if $\mathrm{H}_{2}$ and $L$ have real coefficients then $A$ and $K$ can be chosen to have real coefficients as well.

Notice that the sum

$$
e^{i \hbar^{-1} \operatorname{ad}_{A}}\left(H_{2}+L\right)=\sum_{\ell} \frac{1}{\ell !}\left(\frac{i}{\hbar} \operatorname{ad}_{A}\right)^{\ell}\left(H_{2}+L\right)
$$

is indeed convergent in $\mathscr{E}$ because $\frac{i}{\hbar} \operatorname{ad}_{A}$ sends $\mathscr{O}_{N}$ into $\mathscr{O}_{N+1}$.

Proof. We construct $A$ (and hence $K$ ) by successive approximations with respect to the filtration of $\mathscr{E}$. Modulo $\mathscr{O}_{3}$ the equality is trivial. So let $N \geqslant 1$ and suppose that for some $A_{N} \in \mathscr{O}_{3}$ we have

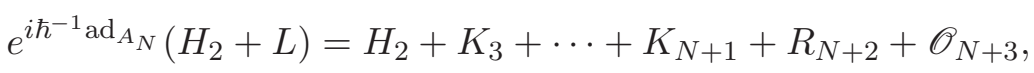

where $K_{i} \in \mathscr{D}_{i}$ and commutes with $H_{2}, R_{N+2} \in \mathscr{D}_{N+2}$. Let $A^{\prime} \in \mathscr{D}_{N+2}$; then a small calculation gives

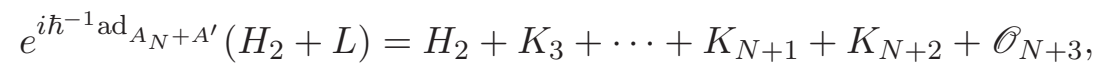


where

$$
K_{N+2}=R_{N+2}+i \hbar^{-1} \operatorname{ad}_{A^{\prime}} H_{2}=R_{N+2}-i \hbar^{-1} \operatorname{ad}_{H_{2}} A^{\prime} .
$$

We look for an $A^{\prime}$ such that $\left[K_{N+2}, H_{2}\right]=0$. This is possible because $H_{2}$ is admissible. Now if we assume that $H_{2}, L$ and $K_{j}, j \leqslant N+1$ are real, then $R_{N+2}$ is real too. Since $\frac{i}{\hbar} \operatorname{ad}_{H_{2}}=\left\{H_{2}, \cdot\right\}$ is a real endomorphism, we have

$$
\mathscr{D}_{N}^{\mathbb{R}}=\operatorname{ker}^{\mathbb{R}}\left(\operatorname{ad}_{H_{2}}\right)+\operatorname{im}^{\mathbb{R}}\left(\operatorname{ad}_{H_{2}}\right) \text {. }
$$

Hence(武) can be solved with real coefficients.

Remark 2.2 If we write the theorem modulo $\hbar$ we recover the classical Birkhoff normal form for Hamiltonians on $\mathbb{R}^{2 n}$. Indeed, let $p$ and $a$ be $C^{\infty}$ functions on $\mathbb{R}^{2 n}$ with Taylor expansion at the origin $H_{2}(x, \xi)+L(x, \xi, 0)$ and $A(x, \xi, 0)$ respectively. Then if $\phi$ denotes the Hamiltonian flow of $a$ at time 1 , we have

$$
p \circ \phi=H_{2}+k
$$

where $k$ has the asymptotic expansion $K(x, \xi, 0)$. Consequently the Poisson bracket of $H_{2}$ and $k$ is flat at the origin.

Remark 2.3 Another way of constructing the quantum Birkhoff normal form would be to start from the classical result and build successively in increasing powers of $\hbar$. This was used by several authors and amounts to follow a different filtration which, in a sense, is less optimal than ours. Nevertheless the result is the same, as for instance in [27].

Remark 2.4 The result presented here is often called the Birkhoff-Gustavson normal form in the mathematical physics literature. Gustavson popularised the idea of Birkhoff in [17] by providing computer programs performing the canonical transformation involved. Moreover, Gustavson added the analysis of the resonant cases, while in his treatise [5], Birkhoff only dealt with the non-resonant situation. Note that Moser had a similar result before Gustavson, in the article 22]. In some sense, the Birkhoff normal form is the Hamiltonian version of the Poincaré-Dulac method [12]. Actually the Poincaré-Dulac normal form is even more general since it allows for the hypothesis of admissibility to be relaxed. Then $\mathrm{H}_{2}$ has to be split into commuting semisimple and nilpotent parts, and the normalisation is performed with respect to the semisimple part. The quantum version is probably much more complicated to analyse, but it would be very interesting to do so.

In this article we will always assume that $H_{2}$ is elliptic : in some canonical coordinates, one can write

$$
H_{2}=\sum_{j=1}^{n} \frac{\nu_{j}}{2}\left(x_{j}^{2}+\xi_{j}^{2}\right) .
$$


In order to understand what kind of formal series $K$ can show up in the Birkhoff normal form, it is crucial to study the kernel of $\operatorname{ad}_{H_{2}}$. The following lemma is elementary and standard.

Lemma 2.5 $\operatorname{ad}_{H_{2}}$ is diagonal on the $\mathbb{C} \llbracket h \rrbracket$-basis $z^{\beta} \bar{z}^{\gamma}$ where $\beta$, $\gamma$ are multiindices in $\mathbb{N}^{n}$ and $z_{j}=x_{j}+i \xi_{j}$, and

$$
\operatorname{ad}_{H_{2}}\left(z^{\beta} \bar{z}^{\gamma}\right)=\langle\beta-\gamma, \nu\rangle z^{\beta} \bar{z}^{\gamma}
$$

We also state and prove the following - maybe less standard — result, which will be one of the tools in the next sections to obtain a pseudo-differential version of the Birkhoff normal form. Let $\mathcal{R}$ be the resonance module

$$
\mathcal{R}:=\left\{\alpha \in \mathbb{Z}^{n}, \quad\langle\alpha, \nu\rangle=0\right\}
$$

and denote by $n-k$ its rank $(k \geqslant 1)$.

Lemma 2.6 There exists a Hamiltonian $\mathbb{T}^{k}$ action on $\mathbb{R}^{2 n}=T^{*} \mathbb{R}^{n}$ such that the space of all power series that commute with $\mathrm{H}_{2}$ is exactly the space of $\mathbb{T}^{k}$-invariant power series:

$$
\operatorname{ker} \operatorname{ad}_{H_{2}}=\mathscr{E}^{\mathbb{T}^{k}}
$$

Proof . Let us use the obvious notation $H_{2}=\frac{1}{2}\left\langle\nu, x^{2}+\xi^{2}\right\rangle$. One can decompose $\mathrm{H}_{2}$ into

$$
H_{2}=\frac{1}{2}\left\langle\ell_{1}, x^{2}+\xi^{2}\right\rangle+\cdots+\frac{1}{2}\left\langle\ell_{k}, x^{2}+\xi^{2}\right\rangle
$$

where $\ell_{j} \in \mathbb{R} . \mathbb{Z}^{n},\left(\ell_{1}, \ldots, \ell_{k}\right)$ are independent, and each Hamiltonian $\frac{1}{2}\left\langle\ell_{j}, x^{2}+\right.$ $\left.\xi^{2}\right\rangle$ has a periodic flow.

To show this, consider the orthogonal complement of the resonance module

$$
\mathcal{R}^{\perp}=\left\{\mu \in \mathbb{Z}^{n}, \quad\langle\alpha, \nu\rangle=0 \Rightarrow\langle\alpha, \mu\rangle=0 \quad \forall \alpha \in \mathbb{Z}^{n}\right\} .
$$

Let $\left(u^{1}, \ldots, u^{k}\right)$ be a $\mathbb{Z}$-basis of $\mathcal{R}^{\perp}$. One can view the $\mathbb{Q}$-module $\mathbb{Q}$. $\mathcal{R}=$ $\mathcal{R} \otimes \mathbb{Q}$ as a $\mathbb{Q}$-vector space, endowed with the $\mathbb{Q}$-scalar product induced by $\langle\cdot, \cdot\rangle$. Then $(\mathcal{R} \otimes \mathbb{Q})^{\perp}=\mathcal{R}^{\perp} \otimes \mathbb{Q}$ and, by density or some algebraic argument, $(\mathcal{R} \otimes \mathbb{R})^{\perp}=\mathcal{R}^{\perp} \otimes \mathbb{R}$. Therefore $\nu \in \mathcal{R}^{\perp} \otimes \mathbb{R}$, so we have $k \geqslant 1$ and one can decompose

$$
\nu=\sum_{j=1}^{k} \lambda_{j} u^{j}, \quad \lambda_{j} \in \mathbb{R} .
$$

We define $\ell_{j}:=\lambda_{j} u^{j}$. Since $u^{j}$ has integer coefficients, it is clear that the Hamiltonian $\frac{1}{2}\left\langle u^{j}, x^{2}+\xi^{2}\right\rangle$ has a periodic flow.

From lemma 2.5 if $\beta, \gamma$ are multi-indices in $\mathbb{N}^{n}$ and $z_{j}=x_{j}+i \xi_{j}$, then

$$
\operatorname{ad}_{H_{2}}\left(z^{\beta} \bar{z}^{\gamma}\right)=\langle\gamma-\beta, \nu\rangle z^{\beta} \bar{z}^{\gamma} .
$$


But if $\gamma-\beta \in \mathcal{R}$, then

$$
\gamma-\beta \in\left((\mathcal{R} \otimes \mathbb{Q})^{\perp}\right)^{\perp}=\left(\mathcal{R}^{\perp} \otimes \mathbb{Q}\right)^{\perp},
$$

Hence

$$
\forall j, \quad\left\langle u_{j}, \gamma-\beta\right\rangle=0 .
$$

In other words $z^{\beta} \bar{z}^{\gamma}$ commutes with each term in the decomposition (8). Therefore any polynomial in ker ad $H_{2}$ commutes with all $\frac{1}{2}\left\langle\ell_{j}, x^{2}+\xi^{2}\right\rangle$ 's, and thus is invariant under the $\mathbb{T}^{k}$ action they generate.

Remark 2.7 Given a point in $\mathbb{R}^{2 n}$, its orbit under the flow of $H_{2}$ is contained in the $\mathbb{T}^{k}$ orbit of that point. Actually a small variant of the proof shows that the inverses of the primitive periods of each periodic Hamiltonian $\frac{1}{2}\left\langle\ell_{j}, x^{2}+\xi^{2}\right\rangle$ possess no resonance relation, and hence the $H_{2}$-orbit is in fact dense in the $\mathbb{T}^{k}$-orbit.

Corollary 2.8 1. If there is no resonance relation (ie. $k=n$ ) then any element of $\mathscr{E}$ commuting with $\mathrm{H}_{2}$ is of the form

$$
K=f\left(x_{1}^{2}+\xi_{1}^{2}, \ldots, x_{n}^{2}+\xi_{n}^{2} ; \hbar\right),
$$

for a formal series $f$ in $n+1$ variables.

2. More generally if we let

$$
r=\inf \left\{|\alpha| ; \quad \alpha \in \mathbb{Z}^{n}, \alpha \neq 0,\langle\alpha, \nu\rangle=0\right\} \quad \in \mathbb{N}^{*} \cup\{\infty\}
$$

then any element of $\mathscr{E}$ commuting with $\mathrm{H}_{2}$ is of the form

$$
K=f_{r}\left(x_{1}^{2}+\xi_{1}^{2}, \ldots, x_{n}^{2}+\xi_{n}^{2} ; \hbar\right)+R_{r},
$$

where $R_{r} \in \mathscr{O}_{r}$ and $f_{r}(u ; \hbar)$ is a polynomial in $(u ; \hbar)$ of degree at most $[(r-1) / 2]$.

Of course this corollary follows even more obviously from lemma 2.5 alone, since the monomials $z^{\alpha} \bar{z}^{\beta}$ with $|\alpha|+|\beta|<r$ will commute with $H_{2}$ only if $\alpha=\beta$. Hence they admit the form $z^{\alpha} \bar{z}^{\alpha}=\prod\left(x_{i}^{2}+\xi_{i}^{2}\right)^{\alpha_{i}}$.

\section{The semiclassical Birkhoff normal form}

The goal of this section is to show how the formal Birkhoff normal form can be transformed into a more usable semiclassical statement involving spectral estimates. To make the proof more transparent, it is enlightening to separate some statements which are independent of the normal form construction, and which we believe have their own interest. 
In all the article we use the following notation. If $P$ is a self-adjoint operator on some Hilbert space, $P$ bounded from below, then the increasing sequence of eigenvalues below the essential spectrum is denoted by $\lambda_{1}^{P} \leqslant$ $\lambda_{2}^{P} \leqslant \cdots \leqslant \lambda_{j}^{P} \leqslant \cdots$. If $I$ is a borelian of $\mathbb{R}$, the spectral projector of $P$ on $I$ is denoted by $\Pi_{I}^{P}$. If $P$ is a semiclassical pseudo-differential operator, then of course $\lambda_{j}^{P}=\lambda_{j}^{P}(\hbar)$ and $\Pi_{I}^{P}=\Pi_{I}^{P}(\hbar)$ also depend on $\hbar$.

\subsection{Semi-excited spectrum and Taylor expansions}

Let $X$ be either a compact manifold of dimension $n$ equipped with a smooth density or $X=\mathbb{R}^{n}$. We will deal with semiclassical pseudo-differential operators on $X$ in the usual sense, as follows. Let $d$ and $m$ be real numbers. When $X=\mathbb{R}^{n}$, let $S^{d}(m)=S^{d}(m, X)$ the set of all families $(a(\cdot ; \hbar))_{\hbar \in(0,1]}$ of functions in $C^{\infty}\left(T^{*} X\right)$ such that

$$
\forall \alpha \in \mathbb{N}^{n}, \quad\left|\partial_{(x, \xi)}^{\alpha} a(x, \xi ; \hbar)\right| \leqslant C_{\alpha} \hbar^{d}\left(1+|x|^{2}+|\xi|^{2}\right)^{\frac{m}{2}},
$$

for some constant $C_{\alpha}>0$, uniformly in $\hbar$. Then $\Psi^{d}(m, X)$ is the set of all (unbounded) linear operators $A$ on $L^{2}(X)$ that are $\hbar$-Weyl quantisations of symbols $a \in S^{d}(m)$ :

$$
(A u)(x)=\left(O p_{\hbar}^{w}(a) u\right)(x)=\frac{1}{(2 \pi \hbar)^{n}} \int_{\mathbb{R}^{2 n}} e^{\frac{i}{\hbar}\langle x-y, \xi\rangle} a\left(\frac{x+y}{2}, \xi ; \hbar\right) u(y)|d y d \xi| .
$$

The number $d$ in (10) is called the order of the operator. Unless specified, it will always be zero here. In case $X$ is a compact manifold with a smooth density, $\Psi^{d}(m, X)$ is the set of operators on $L^{2}(X)$ that are a locally finite sum $P=\sum_{\beta} P_{\beta}+R$, where for each $\beta$ there is a open set $U_{\beta} \subset X$ equipped with a chart $U_{\beta} \rightarrow \mathbb{R}^{n}$ through which $P_{\beta} \in \Psi^{d}\left(m, \mathbb{R}^{n}\right)$, and $R$ is an integral operator whose Schwartz kernel is $\mathscr{O}\left(\hbar^{\infty}\right)$ in the $C^{\infty}$ topology. Thus, if $X$ is a compact riemannian manifold, $\Delta$ the corresponding Laplacian, and $V \in C^{\infty}(X)$, the Schrödinger operator $P=-\frac{\hbar^{2}}{2} \Delta+V$ is a good candidate, of order zero. In case $X=\mathbb{R}^{n}$, the Schrödinger operator is admissible whenever $V$ has at most a polynomial growth.

Let us denote $\Psi(m, X)=\cup_{d \in \mathbb{Z}} \Psi^{d}(m, X), \Psi^{d}(X)=\cup_{m \in \mathbb{Z}} \Psi^{d}(m, X)$, and $\Psi(X)=\cup_{m \in \mathbb{Z}} \Psi(m, X)$. We shall use in this article the standard properties of such pseudo-differential operators. In particular the composition sends $\Psi(m, X) \times \Psi\left(m^{\prime}, X\right)$ to $\Psi\left(m+m^{\prime}, X\right)$. Moreover all $P \in \Psi(0, X)$ are bounded: $L^{2}(X) \rightarrow L^{2}(X)$, uniformly for $0<\hbar \leqslant 1$.

If $P$ has a real-valued Weyl symbol, then it is a symmetric operator on $L^{2}$ with domain $C_{0}^{\infty}(X)$. If its principal symbol is bounded from below then we use the Friedrichs self-adjoint extension, and we will identify $P$ with this extension. Actually, if the Weyl symbol is real and $p$ is elliptic at infinity (i.e. if there exists $m \in \mathbb{R}$ and a constant $C>0$ such that $P \in \Psi(m, X)$ and its principal symbol $p$ satisfies $|p(x, \xi)| \geqslant \frac{1}{C}\left(\|x\|^{2}+\|\xi\|^{2}\right)^{m / 2}$ for $\|x\|^{2}+\|\xi\|^{2} \geqslant$ 
$C$ ), then $P$ is essentially self-adjoint (see for instance [11, proposition 8.5]). But we won't use this result here.

Finally, when $P \in \Psi(m, X)$ is self-adjoint and $f \in C_{0}^{\infty}(\mathbb{R})$, then $f(P) \in$ $\cap_{m^{\prime}}\left(\Psi\left(m^{\prime}, X\right)\right)$. See for instance [11, [23], or [9] for details. In this work all pseudo-differential operators are assumed to admit a classical asymptotic expansion in integer powers of $\hbar$.

Theorem 3.1 Let $P$ and $Q$ be two semiclassical pseudo-differential operators in $\Psi^{0}(X)$ such that

- at $z_{0} \in T^{*} X$, the principal symbols $p$ and $q$ take their minimal value $p\left(z_{0}\right)=q\left(z_{0}\right)=0$, this minimum is reached only at $z_{0}$ and is nondegenerate;

- there exists $E_{\infty}>0$ such that $\left\{p \leqslant E_{\infty}\right\}$ and $\left\{q \leqslant E_{\infty}\right\}$ are compact.

Suppose that, in some local coordinates near $z_{0}$, the total symbols of $P$ and $Q$ admit the same Taylor expansion at $z_{0}$. Then there exists $E_{0}>0, \hbar_{0}>0$ and for each $N$ a constant $C_{N}>0$ such that for all $(\hbar, E) \in\left[0, \hbar_{0}\right] \times\left[0, E_{0}\right]$

$$
\lambda_{j}^{P} \leqslant E \text { or } \lambda_{j}^{Q} \leqslant E \Rightarrow\left|\lambda_{j}^{P}-\lambda_{j}^{Q}\right| \leqslant C_{N}\left(E^{N}+\hbar^{N}\right) .
$$

Before entering the proof of the theorem, we recall an elementary consequence of the minimax theorem.

Lemma 3.2 Let $A$ and $B$ be two self-adjoint operators on $\mathscr{H}$, both bounded from below. Suppose there exists an interval $I=(-\infty, E]$ and $C>0$ such that $\Pi_{I}^{B}(\mathscr{H}) \subset \operatorname{Dom}(A)$ and

$$
\left\|(A-B) \Pi_{I}^{B}\right\| \leqslant C .
$$

Then for all $j$ such that $\lambda_{j}^{B} \leqslant E$ one has

$$
\lambda_{j}^{A} \leqslant \lambda_{j}^{B}+C .
$$

Proof. Let $\lambda_{j}^{B} \leqslant E$ and $\mathcal{F}_{j}^{B}=\Pi_{\left(-\infty, \lambda_{j}^{B}\right]}^{B}(\mathscr{H})$ the eigenspace associated to the eigenvalues below or equal to $\lambda_{j}^{B}$. Let $\phi \in \mathcal{F}_{j}^{B}$, of norm 1 ; by hypothesis one has

$$
\|A \phi-B \phi\| \leqslant C .
$$

Hence by Cauchy-Schwarz $|\langle A \phi, \phi\rangle-\langle B \phi, \phi\rangle| \leqslant C$. Therefore

$$
\langle A \phi, \phi\rangle \leqslant\langle B \phi, \phi\rangle+C \text {. }
$$

Since $\lambda_{j}^{B}=\sup _{\phi \in \mathcal{F}_{j}^{B},\|\phi\|=1}\langle B \phi, \phi\rangle$, one gets

$$
\sup _{\phi \in \mathcal{F}_{j}^{B},\|\phi\|=1}\langle A \phi, \phi\rangle \leqslant \lambda_{j}^{B}+C .
$$


Now since $\mathcal{F}_{j}^{B}$ has dimension $j$, the minimax formula

$$
\lambda_{j}^{A}=\inf _{\mathcal{F} \subset \operatorname{Dom}(A), \operatorname{dim} \mathcal{F}=j}\left(\sup _{\phi \in \mathcal{F},\|\phi\|=1}\langle A \phi, \phi\rangle\right)
$$

implies that $\lambda_{j}^{A} \leqslant \lambda_{j}^{B}+C$.

When dealing with manifolds $X \neq \mathbb{R}^{n}$, we shall need a refinement of the lemma, as follows.

Lemma 3.3 Let $A$ and $B$ be two self-adjoint operators acting respectively on the Hilbert spaces $\mathscr{H}^{\prime}$ and $\mathscr{H}$, both bounded from below. Suppose there exists a bounded operator $U: \mathscr{H} \rightarrow \mathscr{H}^{\prime}$, an interval $I=(-\infty, E]$ and constants $C>0, c \in(0,1)$ such that $U \Pi_{I}^{B}(\mathscr{H}) \subset \operatorname{Dom}(A)$ and

$$
\left\|\left(U^{*} A U-B\right) \Pi_{I}^{B}\right\| \leqslant C
$$

and

$$
\left\|U^{*} U \Pi_{I}^{B}-\Pi_{I}^{B}\right\| \leqslant c
$$

Then for all $j$ such that $\lambda_{j}^{B} \leqslant E$ one has

$$
\lambda_{j}^{A} \leqslant\left(\lambda_{j}^{B}+C\right)\left(1+\frac{c}{1-c}\right) .
$$

Proof. Using the same notation as in the proof of lemma 3.2, we deduce from the first hypothesis that

$$
\langle A U \phi, U \phi\rangle \leqslant\langle B \phi, \phi\rangle+C,
$$

while the second yields

$$
\left|\|U \phi\|^{2}-1\right| \leqslant c
$$

Hence $\|U \phi\|^{2} \geqslant 1-c>0$. Therefore

$$
\frac{\langle A U \phi, U \phi\rangle}{\|U \phi\|^{2}} \leqslant(\langle B \phi, \phi\rangle+C)\left(1+\frac{c}{1-c}\right) .
$$

Moreover $U: \mathcal{F}_{j}^{B} \rightarrow \mathscr{H}^{\prime}$ is injective and hence $\operatorname{dim}\left(U \mathcal{F}_{j}^{B}\right)=j$. We conclude as in the proof of lemma 3.2 .

Finally, for the proof of theorem 3.1 it will be very convenient to use a generalisation of a well-known microlocalisation result of [27 for which, using the above lemmas, we give a new and simple proof. Recall that we say that a pseudo-differential operator $P \in \Psi(X)$ microlocally vanishes at a point $z \in T^{*} X$ when in some local coordinates its full Weyl symbol vanishes at $z$. 
Lemma 3.4 Let $P \in \Psi\left(X^{\prime}\right)$ and $Q \in \Psi(X)$ be self-adjoint semiclassical pseudo-differential operators, with principal symbols $p$ and $q$. Assume there exists a bounded operator $U: L^{2}(X) \rightarrow L^{2}\left(X^{\prime}\right)$, compact subsets $D \subset T^{*} X$, $D^{\prime} \subset T^{*} X^{\prime}$, and an interval $I=(-\infty, E]$ (with $D, D^{\prime}$ and $E$ being independent of $\hbar$ ) such that

1. $p^{-1}(I)$ (respectively $\left.q^{-1}(I)\right)$ is contained in the interior of $D$ (respectively $\left.D^{\prime}\right)$;

2. $U^{*} P U-Q$ and $U^{*} U-$ Id are pseudo-differential operators that microlocally vanish in $D$;

3. $P-U Q U^{*}$ and $U U^{*}-\mathrm{Id}$ are pseudo-differential operators that microlocally vanish in $D^{\prime}$;

Then there exists $\hbar_{0}>0$ and a positive sequence $\left(C_{N}\right)_{N \in \mathbb{N}}$ (depending on $E)$ such that, for all $j$ and $\hbar<\hbar_{0}$ such that $\lambda_{j}^{Q} \leqslant E$ (or $\lambda_{j}^{P} \leqslant E$ ), one has:

$$
\left|\lambda_{j}^{Q}-\lambda_{j}^{P}\right| \leqslant C_{N} \hbar^{N}
$$

Remark 3.5 In most situations $U$ will be a Fourier integral operator. When $X=X^{\prime}$ and $P, Q \in \Psi(0, X)$, the result was proved in [27, proposition 2.2 ], using the Kato distance to handle the spectral perturbation. Our proof here, using the minimax, looks simpler, but the idea is essentially the same. A small additional argument is needed to handle $X \neq X^{\prime}$. We give the full proof here for the sake of completeness.

Proof. First let $E^{\prime}>E$ such that the hypothesis (1.) still holds when $E$ is replaced by $E^{\prime}$.

The hypothesis (1.) ensures that the spectra of $P$ and $Q$ are strictly bounded from below by some positive constant $E_{0}$, independent of $\hbar$. Moreover it is well known that it also implies that the intersections with $I^{\prime}=$ $\left(-\infty, E^{\prime}\right]$ of these spectra are discrete. One can check this as follows.

Let $E_{1}>E^{\prime}$ be independent of $\hbar$ and such that $q^{-1}\left(\left[E_{0}, E_{1}\right]\right)$ is contained in the interior of $D$. Let $f \in C_{0}^{\infty}(\mathbb{R})$ such that

1. $f=1$ on $\left[\lambda_{1}^{Q}, E^{\prime}\right]$;

2. $f=0$ outside of $\left[E_{0}, E_{1}\right]$.

By pseudo-differential functional calculus (see for instance 23, théorème III-11]), $f(Q)$ is a pseudo-differential operator which belongs to the trace class and hence is compact. This entails that the spectral projector of $Q$ onto $\left[E_{0}, E^{\prime}\right]$ is compact as well, thus proving the discreteness of $\operatorname{Sp}(Q)$ in $\left[E_{0}, E^{\prime}\right]$. Of course the same argument applies to $P$. See also [18 for more details. 
What's more, the functional calculus also ensures that $f(Q)$ microlocally vanishes outside $q^{-1}\left(\left[E_{0}, E_{1}\right]\right)$. By symbolic calculus one has

$$
\left\|\left(Q-U^{*} P U\right) f(Q)\right\|=O\left(\hbar^{\infty}\right) .
$$

Therefore $\operatorname{Dom}(Q f(Q))=\operatorname{Dom}\left(U^{*} P U f(Q)\right)$, in the sense of Friedrichs extensions. But for all $u \in \Pi_{I^{\prime}}^{Q}(\mathscr{H}), u=f(Q) u$. Hence $U u \in \operatorname{Dom}(P)$ and

$$
\left\|\left(Q-U^{*} P U\right) u\right\|=\left\|\left(Q-U^{*} P U\right) f(Q) u\right\|=O\left(\hbar^{\infty}\right)\|u\| .
$$

This shows that there exists a positive sequence $\left(C_{N}\right)_{N \in \mathbb{N}}$ such that for all $N,\left\|\left(Q-U^{*} P U\right) \Pi_{I^{\prime}}^{Q}\right\| \leqslant C_{N} \hbar^{N}$.

Similarly, $\left\|\left(U^{*} U-\mathrm{Id}\right) f(Q)\right\|=O\left(\hbar^{\infty}\right)$ and hence

$$
\left\|\left(U^{*} U-\mathrm{Id}\right) \Pi_{I^{\prime}}^{Q}\right\| \leqslant c_{N} \hbar^{N},
$$

for a positive sequence $\left(c_{N}\right)_{N \in \mathbb{N}}$.

Applying now lemma 3.3 we get, for all $j$ such that $\lambda_{j}^{Q} \leqslant E^{\prime}$, the inequality $\lambda_{j}^{P} \leqslant\left(\lambda_{j}^{Q}+C_{N} \hbar^{N}\right)\left(1+\frac{c_{N} \hbar^{N}}{1-c_{N} \hbar^{N}}\right)$. In particular for $\hbar$ small enough we always reach $\lambda_{j}^{P} \leqslant E^{\prime}$ whenever $\lambda_{j}^{Q} \leqslant E$.

Interchanging the roles of $P$ and $Q$ we obtain as well $\left\|\left(P-U Q U^{*}\right) \Pi_{I^{\prime}}^{P}\right\| \leqslant$ $C_{N} \hbar^{N}$ and $\left\|\left(U U^{*}-\mathrm{Id}\right) \Pi_{I^{\prime}}^{P}\right\| \leqslant c_{N} \hbar^{N}$ (with perhaps a modification of $C_{N}$ and $\left.c_{N}\right)$. Hence a new application of lemma 3.3 yields $\lambda_{j}^{Q} \leqslant\left(\lambda_{j}^{P}+C_{N} \hbar^{N}\right)(1+$ $\left.\frac{c_{N} \hbar^{N}}{1-c_{N} \hbar^{N}}\right)$, uniformly for all $j$ such that $\lambda_{j}^{P} \leqslant E^{\prime}$. This shows that

$$
\left|\lambda_{j}^{Q}-\lambda_{j}^{P}\right| \leqslant\left(C_{N}^{\prime}+E^{\prime}\right) \hbar^{N}
$$

as soon as $\lambda_{j}^{Q} \leqslant E$. Swapping again the roles of $P$ and $Q$ we obtain the final result.

Proof of theorem 3.1. The result of the theorem will be denoted as the property $\mathcal{P}\left(P, Q, \hbar_{0}, E_{0}, C_{N}\right)$. It is easy to see that if $\mathcal{P}\left(P, Q, \hbar_{0}, E_{0}, C_{N}\right)$ and $\mathcal{P}\left(Q, R, \hbar_{0}^{\prime}, E_{0}^{\prime}, C_{N}^{\prime}\right)$ hold, then $\mathcal{P}\left(P, R, \hbar_{0}^{\prime \prime}, E_{0}^{\prime \prime}, C_{N}^{\prime \prime}\right)$ will hold with suitably chosen constants $\hbar_{0}^{\prime \prime}, E_{0}^{\prime \prime}, C_{N}^{\prime \prime}$.

1. - We use this transitivity property to microlocalise the problem in a compact subset of $T^{*} X$. Let $\Phi$ be a pseudo-differential operator that is microlocally equal to the identity on a neighbourhood of the compact

$$
D:=\left\{p \leqslant E_{\infty}\right\} \cup\left\{q \leqslant E_{\infty}\right\}
$$

and microlocally vanishes outside a compact of $T^{*} X$. We may assume also that its principal symbol $\varphi$ satisfies $0 \leqslant \varphi \leqslant 1$. Then consider the operator $P^{\prime}=\Phi P+2 E_{\infty}(\operatorname{Id}-\Phi)$. Its principal symbol $p^{\prime}$ satisfies $p^{\prime}=p$ for $p \leqslant E_{\infty}$ 
and $p^{\prime}>E_{\infty}$ as soon as $p>E_{\infty}$. Hence $P^{\prime}$ satisfies the hypothesis of the theorem as well. Using that $P^{\prime}$ is microlocally equal to $P$ on $D$, we apply lemma 3.4 to $P$ and $P^{\prime}$ with $U=$ Id and an energy $E=E_{0}<E_{\infty}$ such that $\{p \leqslant E\}$ is contained in the interior of $D$. Then, since $\hbar^{N} \leqslant E^{N}+\hbar^{N}$, we obtain $\mathcal{P}\left(P, P^{\prime}, \hbar_{0}, E_{0}, C_{N}\right)$. Using the same trick for $Q$, we construct $Q^{\prime}$ with $\mathcal{P}\left(Q, Q^{\prime}, \hbar_{0}^{\prime}, E_{0}^{\prime}, C_{N}^{\prime}\right)$ for new constants $h_{0}^{\prime}, E_{0}^{\prime}, C_{N}^{\prime}$.

Thus by transitivity we are reduced to prove the theorem for $P^{\prime}$ and $Q^{\prime}$.

2.- We compare now $P^{\prime}$ and $Q^{\prime}$. Notice that $R:=P^{\prime}-Q^{\prime}=\Phi(P-Q)$ microlocally vanishes outside a compact subset of $T^{*} X$. By hypothesis the Weyl symbols of $P^{\prime}$ and $Q^{\prime}$ near $z_{0}$ have the same Taylor expansion. Hence the symbol of $R$ is flat at $z_{0}$. By symbolic calculus we can construct a pseudo-differential operator $S_{N}$ such that

$$
R=S_{N}\left(P^{\prime}\right)^{N}+O\left(\hbar^{\infty}\right)
$$

and $S_{N}$, as $R$ does, microlocally vanishes outside a compact of $T^{*} X$. This implies that $S_{N}$ is bounded for $\hbar \leqslant 1$ by a constant independent of $\hbar$.

Hence (11) implies, for all $E>0$ and $\hbar \leqslant 1$, the following estimate

$$
\left\|R \Pi_{[-E, E]}^{P^{\prime}}\right\| \leqslant D_{N}\left(E^{N}+\hbar^{N}\right) .
$$

We claim that there is a positive sequence $\left(C_{N}\right)$ such that

$$
\left\|R \Pi_{(-\infty, E]}^{P^{\prime}}\right\| \leqslant C_{N}\left(E^{N}+\hbar^{N}\right) .
$$

Indeed let $-E_{\min }$ be the bottom of the spectrum of $P^{\prime}$. If $E_{\min } \leqslant E$ then $\Pi_{(-\infty, E]}^{P^{\prime}}=\Pi_{[-E, E]}^{P^{\prime}}$ and the formula follows from (12) with $C_{N}=D_{N}$. If $E_{\min }>E$ then

$$
\left\|R \Pi_{(-\infty, E]}^{P^{\prime}}\right\| \leqslant\left\|R \Pi_{\left[-E_{\min }, E_{\min }\right]}^{P^{\prime}}\right\| \leqslant D_{N}\left(E_{\min }^{N}+\hbar^{N}\right) .
$$

But by Garding's inequality there is a constant $C>0$ such that $E_{\min } \leqslant C \hbar$. Hence

$$
\left\|R \Pi_{(-\infty, E]}^{P^{\prime}}\right\| \leqslant D_{N}\left(C^{N}+1\right) \hbar^{N} \leqslant C_{N}\left(E^{N}+\hbar^{N}\right)
$$

with $C_{N}=D_{N}\left(C^{N}+1\right)$. Thus the claim is proved.

Now let $E_{0}>0$ be such that the spectrum of $P^{\prime}$ is discrete in $\left(-\infty, E_{0}\right]$. Lemma 3.2 ensures us that if $(\hbar, E) \in[0,1] \times\left[0, E_{0}\right]$ then

$$
\lambda_{j}^{P^{\prime}} \leqslant E \Rightarrow \lambda_{j}^{Q^{\prime}} \leqslant \lambda_{j}^{P^{\prime}}+C_{N}\left(E^{N}+\hbar^{N}\right) .
$$

Finally, as in the proof of lemma 3.4, we may interchange the roles of $P^{\prime}$ and $Q^{\prime}$ to obtain $\mathcal{P}\left(P^{\prime}, Q^{\prime}, \hbar_{0}^{\prime}, E_{0}^{\prime}, C_{N}^{\prime}\right)$, for some new positive constants $\hbar_{0}^{\prime}$, $E_{0}^{\prime}$ and $C_{N}^{\prime}$. 


\subsection{Using the formal Birkhoff normal form}

In this section we consider a pseudo-differential operator $P \in \Psi(X)$ fulfilling the hypothesis of theorem 3.1, transform it into a pseudo-differential operator on $\mathbb{R}^{n}$, take its Taylor series at $z_{0}$, apply the formal Birkhoff normal form, and finally construct a new pseudo-differential operator $Q$ commuting with a harmonic oscillator $\mathrm{H}_{2}$. $Q$ is compared with $P$ using lemma 3.4 and theorem 3.1, hence reducing the spectral study of $P$ to that of an effective Hamiltonian on some eigenspace of the harmonic oscillator.

Thus, first of all, we transfer the spectral problem to $\mathbb{R}^{n}$.

Lemma 3.6 Let $P \in \Psi(X)$ satisfy the hypothesis of theorem 3.1 at a point $z_{0} \in T^{*} X$. Then there exists a pseudo-differential operator $Q \in \Psi\left(\mathbb{R}^{n}\right)$ satisfying the hypothesis of theorem 3.1 at the origin $0 \in \mathbb{R}^{2 n}$, some constants $E>0$ and $\hbar_{0}>0$, and a positive sequence $\left(C_{N}\right)_{N \in \mathbb{N}}$ such that, for all $j$ and $\hbar<\hbar_{0}$ such that $\lambda_{j}^{Q} \leqslant E$ (or $\lambda_{j}^{P} \leqslant E$ ), one has:

$$
\left|\lambda_{j}^{Q}-\lambda_{j}^{P}\right| \leqslant C_{N} \hbar^{N}
$$

Moreover there exist local coordinates near $z_{0}$ in which the full Weyl symbol of $P$ is exactly the Weyl symbol of $Q$.

Proof. Let $(x, \xi)$ be canonical coordinates on a neighbourhood $\Omega$ of $z_{0}=$ $\left(x_{0}, \xi_{0}\right)$ coming from local coordinates $x$ on $X$ and let $U=U(\hbar)$ be the integral operator with Schwartz kernel

$$
U(x, y)=\frac{1}{(2 \pi \hbar)^{n}} \int e^{\frac{i}{\hbar}\langle x-y, \xi\rangle} \varphi(y, x, \xi) d \xi
$$

where $x \in X, y \in \mathbb{R}^{n}$ and $\varphi \in C_{0}^{\infty}\left(\mathbb{R}^{n} \times \Omega\right)$ with $\varphi(y, x, \xi) \equiv 1$ in a neighbourhood of $\left(y_{0}, z_{0}\right)$. Then $U: L^{2}\left(\mathbb{R}^{n}\right) \rightarrow L^{2}(X)$ is bounded and $U^{*} P U \in \Psi\left(\mathbb{R}^{n}\right)$ and has, when expressed in the coordinates $(x, \xi)$, the same Weyl symbol as $P$. This follows from the fact that, restricted to $\Omega$ and expressed in these coordinates, $U$ is simply a compactly supported pseudo-differential operator microlocally equal to the identity near the origin. (From a more geometrical viewpoint $U$ is actually a Fourier integral operator associated to the symplectomorphism $T^{*} X \rightarrow \mathbb{R}^{2 n}$ defined by the canonical coordinates $(x, \xi)$ ). Since the principal symbol of $U^{*} P U$ has a local non-degenerate minimum at the origin, an easy pseudo-differential partition of unity will modify $U^{*} P U$ outside a microlocal neighbourhood $D$ of the origin in such a way that its principal symbol will satisfy the global hypothesis of theorem 3.1. Let $Q$ be the modified operator. Then for $E>0$ small enough, the hypothesis of lemma 3.4 are fulfilled (with $D^{\prime}$ identified with $D$ thanks to the local coordinates in $\Omega$ ). This lemma gives exactly the desired spectral result. 
Remark 3.7 We shall not use the fact that $P$ and $Q$ have the same Weyl symbols in some coordinates. We only retain the geometrical fact that their principal and sub-principal symbols are symplectomorphic.

Using the canonical coordinates of $T^{*} \mathbb{R}^{n}$ we introduce the space $\mathscr{E}$ as in section 2. Using a Borel resummation, one can always quantise an element in $L \in \mathscr{E}$ into a pseudo-differential operator in $\Psi\left(\mathbb{R}^{n}\right)$ whose Weyl symbol has a Taylor series giving back the initial series in $\mathscr{E}$. Moreover we can arbitrarily extend the pseudo-differential operator to vanish microlocally far from $z_{0}$. With a slight abuse with respect to the standard notation, we shall in this section denote by $O p^{W}(L)$ such a pseudo-differential operator; and for any $Q \in \Psi\left(\mathbb{R}^{n}\right)$ we denote by $\sigma_{W}(Q) \in \mathscr{E}$ the Taylor series at $z_{0}$ of the Weyl symbol of $Q$.

Let $Q \in \Psi\left(\mathbb{R}^{n}\right)$ satisfy the hypothesis of theorem 3.1 at $z_{0}=0 \in \mathbb{R}^{2 n}$. Consider the Taylor series $[Q]=\sigma_{W}(Q)$. Since $z_{0}$ is a non-degenerate minimum for $p$, one has

$$
[Q]=\hbar H_{0}+H_{2}+L
$$

where $L \in \mathscr{O}(3), H_{2} \in \mathscr{D}_{2}$ is elliptic, and $H_{0} \in \mathbb{R}$ is the value at the origin of the sub-principal symbol of $Q$. Applying the formal Birkhoff normal form of theorem 2.1 we obtain $[A]$ and $[K]$ in $\mathscr{O}_{3}$ such that $e^{i \hbar^{-1} \operatorname{ad}_{[A]}}\left(H_{2}+[L]\right)=$ $H_{2}+[K]$. Consider the operators $A=O p^{W}([A])$ and $K=O p^{W}([K])$ (so that $\sigma_{W}(A)=[A]$ and $\left.\sigma_{W}(K)=[K]\right)$. Now $e^{i \hbar^{-1} A}$ is a Fourier integral operator and by Egorov's theorem $e^{i \hbar^{-1} A} Q e^{-i \hbar^{-1} A} \in \Psi\left(\mathbb{R}^{n}\right)$.

Lemma 3.8 The Taylor expansion at $z_{0}$ of the Weyl symbol of $e^{i \hbar^{-1} A} Q e^{-i \hbar^{-1} A}$ is $\hbar H_{0}+H_{2}+[K]$.

Proof. Since $A$ is bounded $e^{i \hbar^{-1} A} Q e^{-i \hbar^{-1} A}=\exp \left(\operatorname{ad}_{i \hbar^{-1} A}\right) Q$. Expanding the exponential in the right-hand side using Taylor's formula with integral remainder, one gets

$$
e^{i \hbar^{-1} A} Q e^{-i \hbar^{-1} A}=\sum_{j=0}^{N} \frac{1}{j !}\left(\operatorname{ad}_{i \hbar^{-1} A}\right)^{j} Q+\frac{1}{N !}\left(\operatorname{ad}_{i \hbar^{-1} A}\right)^{N+1} R_{N},
$$

where

$$
R_{N}=\int_{0}^{1}(1-t)^{N}\left(\exp \left(\operatorname{tad}_{i \hbar^{-1} A}\right) Q\right) d t .
$$

By definition of the Lie algebra structure of $\mathscr{E}, \sigma_{W}\left(\sum_{j=0}^{N} \frac{1}{j !}\left(\operatorname{ad}_{i \hbar^{-1} A}\right)^{j} Q\right)$ is precisely $\sum_{j=0}^{N} \frac{1}{j !}\left(\operatorname{ad}_{i \hbar^{-1}[A]}\right)^{j}[Q]=\hbar H_{0}+H_{2}+[K]+\mathscr{O}_{N+1}$. Thus we need to prove that $\sigma_{W}\left(\frac{1}{N !}\left(\operatorname{ad}_{i \hbar^{-1} A}\right)^{N+1} R_{N}\right) \in \mathscr{O}_{N+1}$, and for this it suffices to show that the Weyl symbol of $R_{N}$ is bounded near $z_{0}$, uniformly in $\hbar$. Indeed, its Taylor series would then be in $\mathscr{O}_{0}$ and we would conclude using the fact that $\operatorname{ad}_{i \hbar^{-1}[A]}$ sends $\mathscr{O}_{j}$ to $\mathscr{O}_{j+1}$. But by Egorov's theorem, $\exp \left(\operatorname{tad}_{i \hbar^{-1} A}\right) Q$ is a pseudo-differential operator of order zero, uniformly in $t \in[0,1]$. Integrating over $t$ we get that $R_{N}$ is indeed of order zero. 
Proposition 3.9 For any compact $D \subset \mathbb{R}^{2 n}$ containing the origin in its interior, there exists a pseudo-differential operator $K \in \Psi\left(\mathbb{R}^{n}\right)$, microlocally vanishing outside $D$, such that

- $\left[\hat{H}_{2}, K\right]=0$;

- The Weyl symbols of $e^{i \hbar^{-1} A} Q e^{-i \hbar^{-1} A}$ and $\hbar H_{0}+\hat{H}_{2}+K$ have the same Taylor expansion at the origin;

- $\hbar H_{0}+\hat{H}_{2}+K$ satisfies the hypothesis of theorem 3.1 (with $X=\mathbb{R}^{n}$ and $z_{0}=0$ ).

Proof. We use lemma 2.6, ie. the fact that there exists a $\mathbb{T}^{k}$ action on $\mathbb{R}_{\{x, \xi\}}^{2 n}($ with $k \geqslant 1)$ such that

$$
\operatorname{ker} \operatorname{ad}_{H_{2}}=\mathscr{E}^{T^{k}} .
$$

Let $\tilde{K}$ be a compactly supported Borel resummation of $[K]$. Let $\bar{K}$ be the average of $\tilde{K}$ under the $\mathbb{T}^{k}$-action. Then, since $[K] \in \mathscr{E}^{\mathbb{T}^{k}}, \sigma_{W}(\bar{K})=[K]$. Hence $\sigma_{W}(\bar{K}) \in \mathscr{E}^{T^{k}}$ and by Weyl quantisation, $K:=O p^{W}(\bar{K})$ commutes with $\hat{H}_{2}$. (Recall that commutation is preserved by Weyl quantisation here because $H_{2}$ is quadratic.)

Since $[K] \in \mathscr{O}_{3}$, the last point of the proposition is automatically satisfied if one chooses the support of the Borel resummation to be close enough to the origin.

From the proposition, we deduce:

Corollary 3.10 The operators $Q$ and $\hbar H_{0}+\hat{H}_{2}+K$ have equivalent spectra in the sense of theorem 3.1.

Proof. Apply theorem 3.1 to $e^{i \hbar^{-1} A} Q e^{-i \hbar^{-1} A}$ and $\hbar H_{0}+\hat{H}_{2}+K$.

We are now in position to state and prove the quantum Birkhoff normal form, which reduces the spectral analysis of $P$ in the semiclassical regime to that of a reduced Hamiltonian, $K$, acting on some eigenspace of $H_{2}$ of finite dimension (but growing as $\hbar$ decreases or $E$ increases).

Theorem 3.11 Let $P \in \Psi(X)$ be a semiclassical self-adjoint pseudo-differential operator of order zero such that

- at $z_{0} \in T^{*} X$, the principal symbol $p$ takes its minimal value $p\left(z_{0}\right)=0$, this minimum is reached only at $z_{0}$ and is non-degenerate;

- there exists $E_{\infty}>0$ such that $\left\{p \leqslant E_{\infty}\right\}$ is compact.

Let $H_{0}$ be the value at $z_{0}$ of the sub-principal symbol of $P$. Then there exists a harmonic oscillator $\mathrm{H}_{2}$ on $\mathbb{R}^{2 n}$ (ie. an elliptic element of $\mathscr{D}_{2}$ in the terminology of section (6), and for any compact domain $D \subset \mathbb{R}^{2 n}$ containing the origin in its interior there exists a pseudo-differential operator $K \in$ $\Psi\left(\mathbb{R}^{n}\right)$ of order zero such that 
- $\left[K, \hat{H}_{2}\right]=0$;

- $K$ vanishes microlocally outside of $D$;

- $\sigma_{W}(K) \in \mathscr{O}_{3}$,

and for each $\eta>0$ there exists $E_{0}>0, \hbar_{0}>0$ and for each $N$ a constant $C_{N}>0$ such that for all $(\hbar, E) \in\left[0, \hbar_{0}\right] \times\left[0, E_{0}\right]$,

$$
\left(\lambda_{j}^{P} \leqslant E \text { or } \lambda_{j}^{Q} \leqslant E-\hbar H_{0}\right) \Rightarrow\left|\lambda_{j}^{P}-\lambda_{j}^{Q}-\hbar H_{0}\right| \leqslant C_{N}\left(E^{N}+\hbar^{N}\right),
$$

where

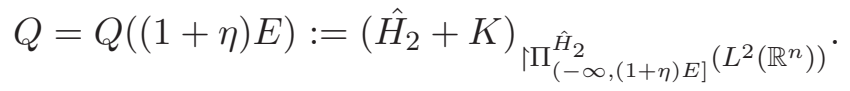

Proof. We first apply lemma 3.6 which allows us to assume that $P \in \Psi\left(\mathbb{R}^{n}\right)$ and $z_{0}=0$.

In view of corollary 3.10 the theorem already holds with $Q((1+\eta) E)$ replaced by $\hat{H}_{2}+K$. But for $E$ and $D$ small enough the spectra of $Q((1+\eta) E)$ and $\hat{H}_{2}+K$ are exactly the same. To see this, we use the following estimate, which is due to the fact that $[K] \in \mathscr{O}_{3}$ :

$$
\exists \hbar_{0}>0, \exists C>0, \quad \forall E^{\prime}>0, \forall \hbar<\hbar_{0} \quad\left\|K \Pi_{\left(-\infty, E^{\prime}\right]}^{\hat{H}_{2}}\right\| \leqslant C E^{\prime \frac{3}{2}} .
$$

This estimate is a particular case of a more general result proven below (lemma 4.2).

Let $E_{c}=4 / 9 C^{2}$ (this is where the function $E^{\prime} \mapsto E^{\prime}-C E^{\prime \frac{3}{2}}$ reaches its maximal value). Then without modifying (14) one can assume that $D$ is included in $H_{2}^{-1}\left(\left(-\infty, E_{c}\right]\right)$ : for this one can replace $K$ by $K f\left(\hat{H}_{2}\right)$ where $f \in C_{0}^{\infty}(\mathbb{R})$ takes values in $[0,1]$, is equal to 1 near the origin, and is supported inside $\left(-\infty, E_{c}\right]$. Then the estimate (14) can be improved as follows:

$$
\forall E^{\prime}>0, \quad\left\|K \Pi_{\left(-\infty, E^{\prime}\right]}^{\hat{H}_{2}}\right\| \leqslant r\left(E^{\prime}\right),
$$

where $r\left(E^{\prime}\right):=\min \left(C E^{\prime \frac{3}{2}}, C E_{c}^{\frac{3}{2}}\right)$. For any $E^{\prime}>0$ and any operator $Q$ we use the notation

$$
\mathscr{H}_{E^{\prime}}^{Q}:=\Pi_{\left(-\infty, E^{\prime}\right]}^{Q}\left(L^{2}(X)\right) .
$$

Also let $Q(\infty):=\hat{H}_{2}+K$. Using that $\mathscr{H}_{E}^{Q(\infty)}$ is stable by $\hat{H}_{2}$ and $\hat{H}_{2}=$ $Q(\infty)-K$ we see that

$$
\mathscr{H}_{E}^{Q(\infty)} \subset \mathscr{H}_{\tilde{E}}^{\hat{H}_{2}},
$$

with $\tilde{E}=E+\left\|K \Pi_{E}^{Q(\infty)}\right\|$. But (16) implies $\left\|K \Pi_{E}^{Q(\infty)}\right\| \leqslant r(\tilde{E})$. Inverting the function $\tilde{E} \mapsto \tilde{E}-r(\tilde{E})$ which by construction is strictly increasing for $\tilde{E} \geqslant 0$ we see that $(16)$ holds as soon as $\tilde{E} \geqslant E+C E^{\frac{3}{2}}+\mathscr{O}\left(E^{2}\right)$, which 
is satisfied if $\tilde{E}=(1+\eta) E$, provided $E$ is small enough. Then (16) says that the eigenvalues of $Q(\infty)$ less than $E$ are the same as the eigenvalues less that $E$ of the restriction of $Q(\infty)$ to $\mathscr{H}_{\tilde{E}}^{\hat{H}_{2}}$. This restriction is precisely $Q((1+\eta) E)$.

\section{The joint spectrum}

In the previous section, we have split $P$ into a harmonic oscillator $\hat{H}_{2}$ and a commuting perturbation $K$ whose microsupport could be arbitrarily small, provided one is only interested in sufficiently small eigenvalues of $P$. As in [27], this can be exploited to describe the so-called semi-excited states, whose energies are of order $\mathscr{O}\left(h^{\gamma}\right), \gamma \in(0,1)$. Here we push the analysis one step further, by introducing a second semiclassical parameter associated to the high energy of the harmonic oscillator $\hat{H}_{2}$ and which turns out to govern the study of the perturbation $K$. In some sense the idea is to have a semiclassical regime associated to the reduction of $K$ by the $\hat{H}_{2}$ action. This view point is made explicit in the next section where we shall assume that this action is periodic. For the moment the game is to control $K$ in a semiclassical regime with two semiclassical parameters...

So, let

$$
H_{2}(x, \xi)=\sum_{j=1}^{n} \frac{\nu_{j}}{2}\left(x_{j}^{2}+\xi_{j}^{2}\right),
$$

defined on $\mathbb{R}^{2 n}$, and let $\hat{H}_{2}$ be its Weyl quantisation, acting on $L^{2}\left(\mathbb{R}^{n}\right)$.

Let $K=K(\hbar) \in \Psi\left(\mathbb{R}^{n}\right)$ a pseudodifferential operator commuting with $\hat{H}_{2}$. Let $\mathscr{H}_{E, \hbar}$ be the eigenspace of $\hat{H}_{2}$ for the eigenvalue $E$. Our goal is to study the restriction of $K$ to $\mathscr{H}_{E, \hbar}$, in terms of the parameters $E$ and $\hbar$, both in a neighbourhood of the origin. More precisely, let $E_{0}>0$ and restrict the set of admissible $E$ 's to eigenvalues of $\hat{H}_{2}$ less that $E_{0}$, ie. we consider the set

$$
\left\{(E, \hbar) \in\left(0, E_{0}\right) \times(0, \infty) / \quad \exists \alpha \in \mathbb{N}^{n}, \quad E=\hbar\left(\frac{|\nu|}{2}+\langle\nu, \alpha\rangle\right)\right\} .
$$

Instead of working with $(E, \hbar)$ we shall use the more convenient scaling $(\epsilon, h)$ defined by

$$
\left\{\begin{array}{l}
\epsilon(E, \hbar)=\sqrt{E} \\
h(E, \hbar)=\hbar / E
\end{array} \quad \epsilon \in\left(0, \epsilon_{0}\right], h^{-1} \in \frac{|\nu|}{2}+\left\langle\nu, \mathbb{N}^{n}\right\rangle\right.
$$

with $\epsilon_{0}=\sqrt{E_{0}}$. Notice that the dimension of $\mathscr{H}_{E, \hbar}$ can be expressed as a function of $h$ alone. Let $\mathscr{H}_{h}=\mathscr{H}_{1, h}$. We identify $\mathscr{H}_{E, \hbar}$ with $\mathscr{H}_{h}$ using the unitary transformation

$$
U_{\epsilon}: \mathscr{H}_{E, \hbar} \rightarrow \mathscr{H}_{h}, \quad f(x) \mapsto \epsilon^{\frac{n}{2}} f(\epsilon x),
$$



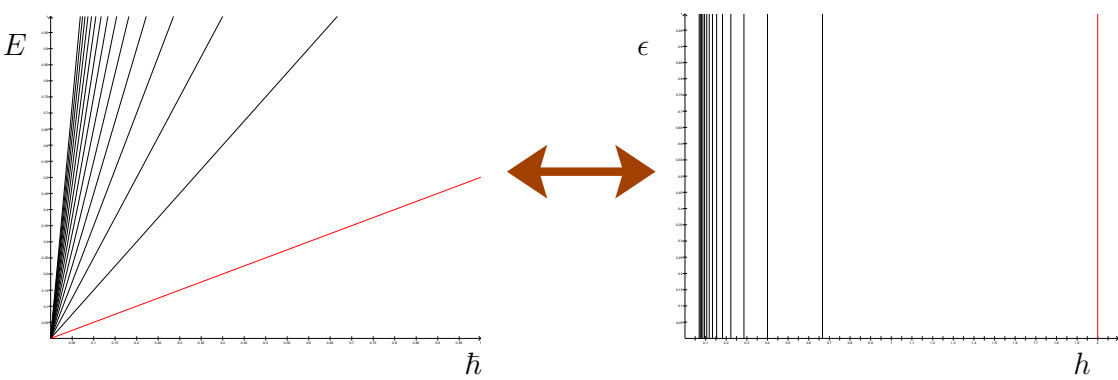

Figure 1: Allowed values for the parameters $(\hbar, E)$ or $(h, \epsilon)$.

The extension of $U_{\epsilon}$ to $L^{2}\left(\mathbb{R}^{n}\right)$ satisfies, for any symbol $p$,

$$
U_{\epsilon} O p_{\hbar}^{w}(p(., \hbar)) U_{\epsilon}^{*}=O p_{h}^{w}(q(., h)),
$$

where $q(x, \xi ; h):=p\left(\epsilon x, \epsilon \xi ; \epsilon^{2} h\right)$.

Definition 4.1 Let $K=K(\hbar)$ be a pseudo-differential operator in $\Psi\left(\mathbb{R}^{n}\right)$. For any positive integers $\ell, m$ we shall say that $K \in \mathscr{M}_{\ell, m}$ if

- its Weyl symbol $k$ is of order $\mathscr{O}\left(\hbar^{m}\right)$ in a neighbourhood of $\left\{H_{2} \leqslant E_{0}\right\}$;

- the Taylor expansion of $k$ at the origin is in $\mathscr{O}_{\ell}$ (with the notation of section 国).

If $K(\hbar)$ commutes with $\hat{H}_{2}$, we denote by $\left(K(\epsilon, h): \mathscr{H}_{h} \rightarrow \mathscr{H}_{h}\right)_{(\epsilon, h)}$ the family of operators defined by

$$
K(\epsilon, h)=U_{\epsilon} K(\hbar)_{\mid \mathscr{H}_{E, \hbar}} U_{\epsilon}^{*} .
$$

The main technical result of this section is the following estimate

Lemma 4.2 Let $K$ be a pseudo-differential operator commuting with $\hat{H}_{2}$. Suppose $K \in \mathscr{M}_{\ell, m}$. Then there exists $C>0, \epsilon_{0}>0$, such that

$$
\|K(\epsilon, h)\| \leqslant C \epsilon^{\ell} h^{m}, \quad \forall \epsilon \in\left(0, \epsilon_{0}\right], \quad \forall h^{-1} \in \frac{|\nu|}{2}+\left\langle\nu, \mathbb{N}^{n}\right\rangle .
$$

To prove this lemma we shall use a specific version of the functional calculus for the harmonic oscillator, which can be obtained as a small improvement of the semiclassical functional calculus of [11]. The only difference is that we allow any value of $h_{0}$, whereas in [11] $h_{0}$ has to be chosen "small enough". This modification is essential for our purposes.

Lemma 4.3 Let $\hat{H}_{2}$ be a harmonic oscillator as in (17). Then for any $h_{0}>0$ and for any $f \in C_{0}^{\infty}(\mathbb{R})$, the family of operators $\left(f\left(\hat{H}_{2}\right)\right)_{h \leqslant h_{0}}$ is a semiclassical pseudo-differential operator in $\Psi(0)$ : there exists a bounded 
family $(a(\cdot, h))_{h \leqslant h_{0}}$ in $S(0)$ such that $f\left(\hat{H}_{2}\right)=O p_{h}^{w}(a(\cdot, h))$. Furthermore, for any integer $m$,

$$
a(\cdot, h)=a_{0}+h a_{1}+h^{2} a_{2}+\cdots+h^{m} r_{m}(\cdot, h)
$$

where all $a_{i}$ are $C_{0}^{\infty}$ with support in the support of $f\left(H_{2}\right)$ (and $a_{0}=f(H)$, $\left.a_{1}=0\right)$, and $\left(r_{k}(\cdot, h)\right)_{h \leqslant h_{0}}$ is a bounded family in $S(-N)$, for all $N \geqslant 0$.

Proof. Assume the result is true for $h_{0}^{\prime}$ and let us prove it for $h_{0}=\delta^{2} h_{0}^{\prime}$. Let $U_{\delta}$ be the unitary map $\left(U_{\delta} \Psi\right)(x)=\delta^{\frac{n}{2}} \Psi(\delta x)$. Since

$$
U_{\delta} \hat{H}_{2}(h) U_{\delta}^{*}=\delta^{2} \hat{H}_{2}\left(h / \delta^{2}\right)
$$

and $U_{\delta}$ is unitary, we have

$$
U_{\delta} f\left(\hat{H}_{2}(h)\right) U_{\delta}^{*}=f\left(\delta^{2} \hat{H}_{2}\left(h / \delta^{2}\right)\right)
$$

By the known result, $f\left(\delta^{2} \hat{H}_{2}\left(h^{\prime}\right)\right)=O p_{h^{\prime}}^{w}\left(b\left(., h^{\prime}\right)\right)$ for $h^{\prime} \leqslant h_{0}^{\prime}$. So

$$
f\left(\hat{H}_{2}(h)\right)=O p_{h}^{w}(a(., h)), \quad \text { for } h \leqslant h_{0}
$$

with $a(x, \xi, h)=b\left(\delta^{-1} x, \delta^{-1} \xi, \delta^{-2} h\right)$. And we deduce the estimates of $a$ from those of $b$.

Proof of lemma 4.2. Let $k(x, \xi ; \hbar)$ be the Weyl symbol of $K$. First (as usual) we can assume that $k$ has compact support. Indeed we split $k=k^{b}+k^{\infty}$, where $k^{b}$ has compact support, $k^{\infty}$ vanishes on

$$
B_{E_{0}+\delta}:=\left\{z ; H_{2}(z) \leqslant E_{0}+\delta\right\}
$$

for some positive $\delta$, and both $k^{b}$ and $k^{\infty}$ commute with $H_{2}$ (using a $\mathbb{T}^{k}$ averaging as in the proof of proposition 3.9). Then we choose $f \in C_{0}^{\infty}(\mathbb{R})$ with value 1 on $\left[0, E_{0}\right]$ and support inside $\left(-\infty, E_{0}+\delta\right)$, so that $f\left(\hat{H}_{2}\right)$ and $K^{\infty}:=O p_{\hbar}^{w}\left(k^{\infty}\right)$ have disjoint microsupport. Then, by lemma 4.3 and symbolic calculus, $f\left(\hat{H}_{2}\right) K^{\infty}=O\left(\hbar^{\infty}\right)$ uniformly for $\hbar \leqslant 1$. Since $\hbar=h \epsilon^{2}, K^{\infty}(\epsilon, h)$ is of order $\mathscr{O}\left(h^{N} \epsilon^{N}\right)$ for all $N$, provided $h \epsilon^{2} \leqslant 2 E_{0} /|\nu|$. Now consider $q(z ; \epsilon, h):=k^{b}\left(\epsilon z ; \epsilon^{2} h\right)$. Since $k^{b}$ has compact support and its Taylor expansion is in $\mathscr{O}_{\ell}$, we have

$$
q=\sum_{2 \alpha+\beta=\ell} \mathscr{O}\left(\left(\epsilon^{2} h\right)^{\alpha}|\epsilon z|^{\beta}\right)=\epsilon^{\ell} \sum_{2 \alpha+\beta=\ell} \mathscr{O}\left(h^{\alpha}|z|^{\beta}\right) .
$$

Since $k^{b}=\mathscr{O}\left(\hbar^{m}\right)$ we know that we can actually write

$$
q=\epsilon^{\ell} h^{m} \sum_{2 \alpha^{\prime}+\beta=\ell-2 m} \mathscr{O}\left(h^{\alpha^{\prime}}|z|^{\beta}\right) .
$$

We could conclude here by restricting to a compact subset in the new variable $z$ and using standard semiclassical arguments similar to what we 
already used at several places. However this would require to restrict the validity of the expected estimate to sufficiently small values of $h$. So we need to refine the analysis.

By hypothesis $h$ is bounded (by $2 /|\nu|$ ); so there is a constant $C>0$ (depending of course on $\ell, m, \nu$ ) such that

$$
|q(z ; \epsilon, h)| \leqslant C \epsilon^{\ell} h^{m}\langle z\rangle^{\ell-2 m},
$$

For all multi-indices $\alpha \in \mathbb{N}^{2 n}, \partial_{z}^{\alpha} k^{b}$ still has compact support and its Taylor expansion is in $\mathscr{O}_{\ell-|\alpha|}$. So we see similarly that for any $\alpha$,

$$
\left|\partial_{z}^{\alpha} q(z ; \epsilon, h)\right| \leqslant C_{\alpha} \epsilon^{\ell} h^{m}\langle z\rangle^{\ell-2 m-|\alpha|} .
$$

In particular $\left(h^{-m} \epsilon^{-\ell} O p_{h}^{w}(q)\right) \in S(\ell-2 m)$, uniformly for all $\epsilon \leqslant \epsilon_{0}, h \leqslant$ $2 /|\nu|$. Using lemma 4.3 with $h_{0}=2 /|\nu|$ and a function $f \in C_{0}^{\infty}(\mathbb{R})$, we consider the operator

$$
\tilde{Q}:=h^{-m} \epsilon^{-\ell} O p_{h}^{w}(q) f\left(O p_{h}^{w}\left(H_{2}\right)\right) .
$$

By the composition theorem for pseudo-differential operators (11], proposition 7.7]), $\tilde{Q}$ is uniformly in $S(0)$, and hence bounded.

It remains to recall that $K(\epsilon, h)=O p_{h}^{w}(q)_{\left.\right|_{\mathscr{H}_{h}}}$ which says that

$$
K(\epsilon, h)=h^{m} \epsilon^{\ell} f(1) \tilde{Q},
$$

and gives the result.

Let us now turn to some applications of our results.

\subsection{Polynomial differential operators}

If $k \in \mathscr{D}_{i}$ then $O p_{\hbar}^{w}(k)$ is a $\hbar$-differential operator with polynomial coefficients. Using (20) we see that

$$
U_{\epsilon} O p_{\hbar}^{w}(k) U_{\epsilon}^{*}=\epsilon^{i} Q(h),
$$

where $Q(h)$ is a $h$-differential operator with polynomial coefficients, independent of $\epsilon$, of order at most $i$ in $\left(x, \partial_{x}\right)$.

Now suppose $K$ is a $\hbar$-pseudo-differential operator commuting with $\hat{H}_{2}$, with formal Weyl symbol $[K]$. Fix $\ell \geqslant 0$ and write

$$
[K]=k^{(0)}+k^{(1)}+\cdots+k^{(\ell)}+r_{\ell+1},
$$

with $k^{(i)} \in \mathscr{D}_{i}$ and $r_{\ell+1} \in \mathscr{O}(\ell+1)$. Applying lemma 4.2, we get

$$
K(\epsilon, h)=K_{0}(h)+\epsilon K_{1}(h)+\cdots+\epsilon^{\ell} K_{\ell}(h)+\epsilon^{\ell+1} R_{\ell+1}(h),
$$


where $R_{\ell+1}(h)$ is uniformly bounded with respect to $h$ and $K_{i}(h)=O p_{h}^{w}\left(k^{(i)}\right)$ (actually, with some abuse of notation, we have written $K_{i}(h)$ for their restrictions to $\mathscr{H}_{h}$ ).

In other words, in order to study the spectrum of $K(\epsilon, h)$, we can restrict to the study of polynomial differential operators commuting with $\mathrm{H}_{2}$, up to an error of order $\mathscr{O}\left(\epsilon^{\ell+1}\right)$, uniformly in $h$. This is very important in practice for numerical calculations, since the matrix elements of such $K_{i}$ 's on a basis of eigenvectors of the harmonic oscillator are very easy to compute (especially when the $K_{i}$ 's are written in terms of creation an annihilation operators or - this amounts to the same - when studied in the BargmannFock representation). See [10] for the case of the 1:2 resonance.

We state the result with the original parameters $(E, \hbar)$ for further reference.

Theorem 4.4 Let $P$ be a $\hbar$-pseudo-differential operator satisfying the hypothesis of theorem 3.11. Then there exists a harmonic oscillator $\mathrm{H}_{2}$ on $\mathbb{R}^{2 n}$ and a formal series

$$
[K]=k^{(3)}+k^{(4)}+\cdots \in \mathscr{O}_{3}
$$

commuting with $\mathrm{H}_{2}$ such that, for any $\ell \in \mathbb{N}$ and any $\eta>0$, there exists $E_{0}>0, \hbar_{0}>0, C>0$ such that for all $E \in\left(0, E_{0}\right], \hbar \in\left(0, \hbar_{0}\right]$ and $j \in \mathbb{N}$,

$$
\left(\lambda_{j}^{P} \leqslant E \text { or } \lambda_{j}^{Q_{\ell}} \leqslant E\right) \Rightarrow\left|\lambda_{j}^{P}-\lambda_{j}^{Q_{\ell}}-\hbar H_{0}\right| \leqslant C E^{\frac{\ell+1}{2}}+\mathscr{O}\left(\hbar^{\infty}\right),
$$

where

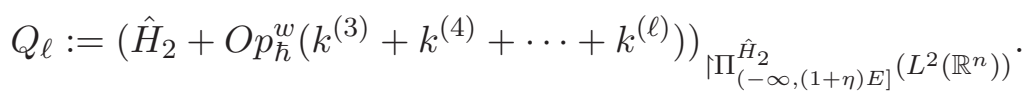

Proof. Naturally, we first apply theorem 3.11. Therefore the question is reduced to the determination of the spectrum of the operator $Q((1+\eta) E)$ defined in that theorem, up to an error of order $\mathscr{O}\left(E^{\infty}\right)+\mathscr{O}\left(\hbar^{\infty}\right)$. We need to compare $Q((1+\eta) E)$ to $Q^{\ell}$. But equation (24) yields

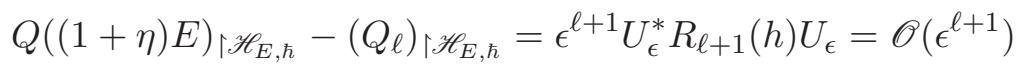

with $\epsilon=\sqrt{E}$. Hence $Q((1+\eta) E)-Q_{\ell}=\mathscr{O}((1+\eta) E)^{\frac{\ell+1}{2}}=\mathscr{O}\left(E^{\frac{\ell+1}{2}}\right)$. The minimax (lemma 3.2) gives the result.

\subsection{The semi-excited Weyl law}

Let $P$ be a $\hbar$-pseudo-differential operator satisfying the hypothesis of theorem 3.11, and let $\mathrm{H}_{2}$ be the corresponding harmonic oscillator, as in (17). We define the resonance order of $H_{2}$ to be

$$
r_{\nu}:=\max \left(3, \inf \left\{|\alpha| ; \quad \alpha \in \mathbb{Z}^{n}, \alpha \neq 0,\langle\alpha, \nu\rangle=0\right\}\right) \in \mathbb{N} \cup\{\infty\} .
$$


By convention $r_{\nu}=\infty$ is $\nu$ is non resonant. Notice that, in contrast to corollary 2.8, we exclude here the value $r_{\nu}=2$ since we shall always deal with perturbations terms that are formally in $\mathscr{O}_{3}$. This remark pertains through the remaining of the article.

We are interested here in the counting function

$$
\mathscr{N}^{P}(E, \hbar):=\#\left\{j ; \quad \lambda_{j}^{P}(\hbar) \leqslant E\right\} .
$$

Theorem 4.5 Let $p$ be the principal symbol of $P$. For any $\ell<r_{\nu}$ (and $\ell \geqslant 2$ )

$$
\mathscr{N}^{P}(E, \hbar)=\frac{1}{(2 \pi \hbar)^{n}} \int_{p \leqslant E}|d x d \xi|+\mathscr{O}\left(\hbar^{-n} E^{n-1}\left(\hbar+E^{\frac{\ell+1}{2}}\right)\right)
$$

uniformly for $E$ and $\hbar$ small enough.

Corollary 4.6 For any $C>0, \gamma>0, \ell \in\left[2, r_{\nu}\right)$ the Weyl counting function for "semi-excited states" has the following asymptotics, as $\hbar \rightarrow 0$ :

$$
\mathscr{N}^{P}\left(C \hbar^{\gamma} ; \hbar\right)=\frac{1}{(2 \pi \hbar)^{n}} \int_{p \leqslant E}|d x d \xi|+\mathscr{O}\left(\hbar^{(1-n)(1-\gamma)}\left(1+\hbar^{\gamma \frac{\ell+1}{2}-1}\right)\right) .
$$

In other words if $\gamma \leqslant \frac{2}{\ell+1}$ then the remainder is $\mathscr{O}\left(\hbar^{-n+\gamma\left(n+\frac{\ell-1}{2}\right)}\right)$ while if $\gamma \geqslant \frac{2}{\ell+1}$ the remainder is $\mathscr{O}\left(\hbar^{(1-n)(1-\gamma)}\right)$. We see that if $\gamma<\frac{2}{r_{\nu}+1}$ then one always gets the best remainder $\mathscr{O}\left(\hbar^{(1-n)(1-\gamma)}\right)$.

Proof of theorem 4.5. Apply theorem 4.4 with the same $\ell$. Then, using the notation of that theorem,

$$
\mathscr{N}^{P}(E, \hbar)=\mathscr{N}^{Q_{\ell}}(E, \hbar)+r(E, \hbar),
$$

where $r(E, \hbar) \leqslant \#\left\{j ; \quad \lambda_{j}^{Q_{\ell}} \in\left[E-C E^{\frac{\ell+1}{2}}, E+C E^{\frac{\ell+1}{2}}\right]\right\}$. Since $\ell<r_{\nu}$ we can see using corollary 2.8 that

$$
Q_{\ell}=f\left(\frac{x_{1}^{2}+\hat{\xi}_{1}^{2}}{2}, \ldots, \frac{x_{n}^{2}+\hat{\xi}_{n}^{2}}{2} ; \hbar\right),
$$

where $f(u ; \hbar)$ is a polynomial in $(u, \hbar)$ of degree at most $[\ell / 2]$, with linear part equal to $\langle u, \nu\rangle$. The eigenvalues of $Q_{\ell}$ are therefore of the form

$$
\lambda_{j}^{Q_{\ell}}=f\left(\hbar\left(\frac{1}{2}+\alpha_{1}\right), \ldots, \hbar\left(\frac{1}{2}+\alpha_{n}\right)\right)
$$

for integers $\left(\alpha_{1}, \ldots, \alpha_{n}\right) \in \mathbb{N}^{n}$, and

$$
\begin{gathered}
\mathscr{N}^{Q_{\ell}}\left(E_{1}, E_{2} ; \hbar\right):=\#\left\{j ; \quad \lambda_{j}^{Q_{\ell}} \in\left[E_{1}, E_{2}\right]\right\} \\
=\#\left\{\alpha \in \mathbb{N}^{n} ; \quad f\left(\hbar\left(\frac{1}{2}+\alpha_{1}\right), \ldots, \hbar\left(\frac{1}{2}+\alpha_{n}\right)\right) \in\left[E_{1}, E_{2}\right]\right\} .
\end{gathered}
$$


Using a covering of $\mathbb{R}^{n}$ by small cubes with sides of length $2 \hbar$, centred at $\left(\hbar\left(\frac{1}{2}+\alpha_{1}\right), \ldots, \hbar\left(\frac{1}{2}+\alpha_{n}\right)\right), \alpha \in \mathbb{Z}^{n}$, it is then easy to see that

$$
\begin{gathered}
\hbar^{n} \mathscr{N}^{Q_{\ell}}\left(E_{1}, E_{2} ; \hbar\right)=\operatorname{Vol}\left(f^{-1}\left(\left[E_{1}, E_{2}\right]\right)\right) \\
+\mathscr{O}\left(\operatorname{Vol}\left(f^{-1}\left(\left[E_{1}-\hbar, E_{1}+\hbar\right]\right)\right)\right)+\mathscr{O}\left(\operatorname{Vol}\left(f^{-1}\left(\left[E_{2}-\hbar, E_{2}+\hbar\right]\right)\right)\right)
\end{gathered}
$$

For $E$ small enough, using that $f(u ; \hbar) \sim\langle u, \nu\rangle$ with $\nu_{i}>0$, one has the bound

$$
\operatorname{Vol}\left(f^{-1}([E-\delta, E+\delta])\right)=\mathscr{O}\left(\delta E^{n-1}\right) .
$$

Applying this with $\delta=\hbar$ and $\delta=C E^{\frac{\ell+1}{2}}$ we get from (26)

$$
\mathscr{N}^{Q_{\ell}}\left(E-C E^{\frac{\ell+1}{2}}, E+C E^{\frac{\ell+1}{2}}\right)=\mathscr{O}\left(\hbar^{-n} E^{n-1}\left(\hbar+E^{\frac{\ell+1}{2}}\right)\right) .
$$

Another application of (26), with $E_{1}=0, E_{2}=E$, combined with (25), yields:

$$
\mathscr{N}^{P}(E ; \hbar)=\hbar^{-n} \operatorname{Vol}\{f \leqslant E\}+\mathscr{O}\left(\hbar^{-n} E^{n-1}\left(\hbar+E^{\frac{\ell+1}{2}}\right)\right) .
$$

Notice now that, by a simple Fubini argument,

$$
\operatorname{Vol}\{f \leqslant E\}=(2 \pi)^{-n} \int_{q^{\ell} \leqslant E}|d x d \xi|,
$$

where $q^{\ell}$ is the full symbol of $Q_{\ell}$. We know from lemma 3.6 and proposition 3.9 that $p=q^{\ell}+\mathscr{O}_{\ell+1}+\mathscr{O}(\hbar)$. Therefore

$$
\begin{aligned}
& \quad\left\{q^{\ell} \leqslant E-C^{\prime}\left(\hbar+E^{\frac{\ell+1}{2}}\right)\right\} \subset\{p \leqslant E\} \subset\left\{q^{\ell} \leqslant E+C^{\prime}\left(\hbar+E^{\frac{\ell+1}{2}}\right)\right\}, \\
& \text { so } \int_{q^{\ell} \leqslant E}|d x d \xi|=\int_{p \leqslant E}|d x d \xi|+\mathscr{O}\left(E^{n-1}\left(\hbar+E^{\frac{\ell-1}{2}}\right)\right) .
\end{aligned}
$$

\subsection{The low-lying eigenvalues}

Let $P$ be a $\hbar$-pseudo-differential operator satisfying the hypothesis of theorem 3.11, and let $\mathrm{H}_{2}$ be the corresponding harmonic oscillator, as in (17). For an energy $E$ of order $\hbar$, the Weyl formula (corollary 4.6) says that the number of eigenvalues of $P$ below $E$ is bounded, independently of $\hbar$. Of course, this can be obtained directly as a consequence of theorem 4.4: because for $E=C \hbar$, the dimension of $\Pi_{(-\infty,(1+\eta) E]}^{\hat{H}_{2}}\left(L^{2}\left(\mathbb{R}^{n}\right)\right)$ is independent of $\hbar$. We use here the Birkhoff normal form to recover a result of Helffer-Sjöstrand [19, theorem 3.6] concerning the asymptotics of the lowlying eigenvalues of Schrödinger operators.

Theorem 4.7 For any $C>0$ and $\hbar$ small enough, the spectrum of $P$ in $(-\infty, C \hbar]$ consists of a finite number, independent of $\hbar$, of eigenvalues. These eigenvalues admit an asymptotic expansion of the form

$$
\lambda_{j}^{P}(\hbar) \sim \hbar H_{0}+\hbar \mu_{0}+\hbar^{\frac{3}{2}} \sum_{m=0}^{\infty} \hbar^{\frac{m}{2}} \mu_{m}
$$


where $\hbar \mu_{0}$ is an eigenvalue of $\hat{H}_{2}$, and, as usual, $H_{0}$ is the value of the subprincipal symbol of $P$ at the minimum of its principal symbol. The number of eigenvalues with a given $\mu_{0}$ is equal for $\hbar$ small enough to the multiplicity of $\hbar \mu_{0}$ for $\hat{H}_{2}$. In particular the smallest eigenvalue of $P$ is simple for $\hbar$ small enough.

Proof. We apply theorem 4.4 with $E=C \hbar$ and $\ell$ large. Thus, modulo an error of size $\mathscr{O}\left(\hbar^{(\ell+1) / 2}\right)$, the eigenvalues of $P$ less than $C \hbar$ are equal to the eigenvalues of $Q_{\ell}+\hbar H_{0}$. Decomposing the Hilbert space on which $Q_{\ell}$ acts according to the eigenspaces of the harmonic oscillator $\hat{H}_{2}$, and using the unitary equivalence as in (23), we are reduced to the study of eigenvalues of matrices of the form

$$
Q^{\ell}(\epsilon, h)=\epsilon^{2} K_{2}(h)+\epsilon^{3} K_{3}(h)+\cdots+\epsilon^{\ell} K_{\ell}(h),
$$

acting on $\mathscr{H}_{h}$, where $h^{-1}=\frac{|\nu|}{2}+\langle\alpha, \nu\rangle$ for some fixed $\alpha \in \mathbb{N}^{n}$ and $\epsilon=\sqrt{\hbar h^{-1}}$. By standard perturbation theory for matrices, the spectrum of $\epsilon^{-2} Q^{\ell}(\epsilon, h)$ is analytic in $\epsilon$ for small $\epsilon$. Since $\epsilon^{2} K_{2}(h)$ is unitarily equivalent to $\hat{H}_{2}$ we obtain the expansion (27), along with the statement concerning the multiplicities.

Remark 4.8 In case $P$ is a semiclassical Schrödinger operator $-\frac{\hbar^{2}}{2} \Delta+V(x)$, This result appeared almost simultaneously in [26, theorem 5.1] and [19, theorem 3.6]. The techniques of [19] could in principle be easily generalised to treat, like we do here, general pseudo-differential operators. While these results are now well known, is it interesting to remark that the appearance of half-integer powers of $\hbar$ in the asymptotic expansion was not so obvious at that time. Actually in [26] these half-integer powers had been forgotten.

The Birkhoff normal form we used here makes it very clear as to why and when such powers may appear in the asymptotic expansion of the eigenvalues. In particular the smallest exponent from which such half-integer powers can appear is half the resonance order $r_{\nu}$. Indeed, below this order, corollary 2.8 shows that the operators $K_{j}(h)$ must be of the form $f_{j}\left(\frac{x_{1}^{2}+\hat{\xi}_{1}^{2}}{2}, \cdots, \frac{x_{n}^{2}+\hat{\xi}_{n}^{2}}{2}\right)$ for some polynomial $f_{j}$ and hence have even order in $(x, \xi)$. Notice that in dimension $1, r_{\nu}=\infty$; hence only integer powers of $\hbar$ may show up in that case. As remarked in [19], the simplest case where half-integer powers of $\hbar$ are present is the so-called $1: 2$ resonance: $n=2$ and $\nu=(1,2)$. The coefficient of $\hbar^{3 / 2}$ is then the average along the flow of $H_{2}$ of the term of order 3 in the Taylor expansion of the symbol. A more general statement is given in section 5.3.

\section{Toeplitz operators}

As we saw in equation (13), our initial spectral problem is reduced to the spectral analysis of a pseudo-differential operator invariant under a $\mathbb{T}^{k}$ ac- 
tion, for some $k=1, \ldots, n$. With this respect, we have two qualitatively extreme situations: $k=n$ and $k=1$. In the first case $k=n$ the harmonic oscillator $\mathrm{H}_{2}$ has no resonance relation, and the situation is essentially completely integrable, at least in the semi-excited regime. This can be seen explicitly with theorem 4.4, in view of corollary 2.8.

On the contrary, in the case $k=1$, the harmonic oscillator $H_{2}$ is completely resonant: up to a common multiple, all frequencies $\nu_{i}$ are integers. The $\mathbb{T}^{1}$ action is precisely the flow of $H_{2}$. From the perspective of integrability, the spectral analysis looks more involved. However the fact that $\mathrm{H}_{2}$ has a periodic flow forces the spectrum to split into regularly spaced clusters. The spectral analysis becomes in some sense simpler, since it comes down to the study of each individual cluster. From a geometric point of view each of these clusters correspond to the spectrum of an operator acting on a reduced space. The goal of this section is to develop this idea.

In principle, the mixed case $1<k<n$ could be treated by a combination of both techniques. But this still has to be investigated further.

We use the notation introduced in the beginning of section 4 In particular we deal with rescaled parameters $\epsilon, h$ and, using the scaling operator $U_{\epsilon}$ as in (19), we are able to reduce the analysis to the case $E=1$. The new tool we introduce here is to work in the Bargmann space $\mathscr{B}_{h}$ [ [4]. Recall that $\mathscr{B}_{h}$ is the space of entire holomorphic functions on $\mathbb{C}^{n}$ with finite norm, where the norm comes from the scalar product

$$
\left\langle\psi, \psi^{\prime}\right\rangle_{\mathbb{C}^{n}}=\int_{\mathbb{C}^{n}}\left(\psi, \psi^{\prime}\right)(z) \mu(z), \quad \text { with }\left(\psi, \psi^{\prime}\right)(z)=\psi(z) \overline{\psi^{\prime}(z)} e^{-|z|^{2} / h}
$$

where $|z|^{2}=\sum z_{i} \bar{z}_{i}$ and $\mu$ is the Lebesgue measure on $\mathbb{C}^{n}=\mathbb{R}^{2 n}$. Operators on $L^{2}\left(\mathbb{R}^{n}\right)$ can be transported on $\mathscr{B}_{h}$ via the Bargmann transform which is the unitary map $U_{\mathscr{B}}: L^{2}\left(\mathbb{R}^{n}\right) \rightarrow \mathscr{B}_{h}$ given by

$$
U_{\mathscr{B}}(\varphi)(z)=\frac{2^{n / 4}}{(2 \pi h)^{3 n / 4}} \int_{\mathbb{R}^{n}} e^{h^{-1}\left(z \cdot x \sqrt{2}-\left(z^{2}+x^{2}\right) / 2\right)} \varphi(x) d x,
$$

where $z \cdot x=\sum z_{i} x_{i}, z^{2}=z \cdot z, x^{2}=x \cdot x$.

The harmonic oscillator $\hat{H}_{2}(h):=O p_{h}^{w}\left(H_{2}\right)$ with $H_{2}$ as in (17) becomes

$$
\hat{H}_{2}^{\mathscr{B}}(h):=U_{\mathscr{B}} \tilde{H}_{2}(h) U_{\mathscr{B}}^{*}=h \sum_{j=1}^{n} \nu_{j}\left(z_{j} \frac{\partial}{\partial z_{j}}+\frac{1}{2}\right) .
$$

In order to deal with symbols of operators in the Bargmann side we simply identify the real phase space $\mathbb{R}^{2 n}$ with $\mathbb{C}^{n}$ using $z_{j}=\left(x_{j}-i \xi_{j}\right) / \sqrt{2}$. Hence $H_{2}=\sum_{j} \nu_{j}\left|z_{j}\right|^{2}$. 


\subsection{The reduction setting}

In all this section 5 the main assumption is that for all $i, j, \nu_{i} / \nu_{j} \in \mathbb{Q}$. Then there exists positive coprime integers $\mathfrak{p}_{1}, \ldots, \mathfrak{p}_{n}$, and a constant $\nu_{c}>0$ such that $\nu_{j}=\nu_{c} \mathfrak{p}_{j}$. Hence, according to (18), our rescaled semiclassical parameter $h$ is of the form

$$
h^{-1}=\frac{|\nu|}{2}+\nu_{c} N, \quad N \in \mathbb{N} .
$$

Let $Y=\left\{y \in \mathbb{C}^{n} ; \quad H_{2}(y)=1\right\} ;$ it is a smooth, compact submanifold of $\mathbb{C}^{n}$. The Hamiltonian flow of $H_{2}$ defines a locally free action of $S^{1}$ on $Y$

$$
S^{1} \times Y \rightarrow Y, \quad(u, y) \mapsto u . y:=\left(u^{\mathfrak{p}_{1}} y_{1}, \ldots, u^{\mathfrak{p}_{n}} y_{n}\right)
$$

where we identify $S^{1}$ with the complex numbers of modulus 1 .

The quotient $Y / S^{1}$ is an orbifold $M$. It is endowed with the MarsdenWeinstein symplectic form $\omega$, naturally defined by $\pi^{*} \omega=\omega_{Y}$ where $\pi$ is the projection $Y \rightarrow M$ and $\omega_{Y}$ is the restriction to $Y$ of the canonical symplectic form of $\mathbb{C}^{n}$. Smooth functions on $M$ are by definition $S^{1}$-invariant functions on $Y$.

Let $C_{N}^{\infty}(Y) \subset C^{\infty}(Y)$ be the space of equivariant functions in the following sense:

$$
\psi(u . y)=u^{N} \psi(y), \quad \forall u \in S^{1}, y \in Y .
$$

$M$ is naturally endowed with a complex line bundle $L \rightarrow Y$ whose sections are identified with functions of $C_{1}^{\infty}(Y)$ using the pull-back $\pi^{*}$. More generally $C^{\infty}\left(M, L^{N}\right) \simeq C_{N}^{\infty}(Y)$. Let us endow $L$ with the hermitian structure such that, if $\psi_{r}$ and $\psi_{r}^{\prime}$ are sections of $L^{N}$ and $\psi=\pi^{*} \psi_{r}, \psi^{\prime}=\pi^{*} \psi_{r}^{\prime}$, then

$$
\left(\psi_{r}, \psi_{r}^{\prime}\right)(\pi(y))=\psi(y) \overline{\psi^{\prime}(y)} e^{-\nu_{c} N|y|^{2}} .
$$

This defines the scalar product $\left\langle\psi_{r}, \psi_{r}^{\prime}\right\rangle_{M}:=\int_{M}\left(\psi_{r}, \psi_{r}^{\prime}\right) \mu_{M}$, where $\mu_{M}$ is the Liouville (or symplectic) measure of $M$.

On the other hand let $\mathscr{H}_{h}^{\mathscr{B}}=\operatorname{ker}\left(\hat{H}_{2}^{\mathscr{B}}(h)-1\right)=U_{\mathscr{B}} \mathscr{H}_{h}$ where $\mathscr{H}_{h}$ is, as

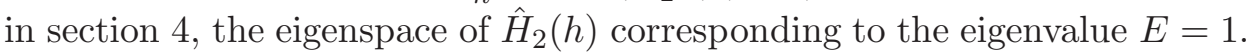
It is well known [4] that the monomials $z^{\alpha}=z_{1}^{\alpha_{1}} \cdots z_{n}^{\alpha_{n}}$ such that

$$
\langle\mathfrak{p}, \alpha\rangle=N
$$

form a basis of $\mathscr{H}_{h}^{\mathscr{B}}$. But equation (31) also shows that the restrictions of the $z^{\alpha}$ 's to $Y$ belong to $C_{N}^{\infty}(Y)$. Projecting onto $M$ we thus see that $\mathscr{H}_{h}^{\mathscr{B}}$ may be identified with a subspace $\mathbf{H}_{N} \subset C^{\infty}\left(M, L^{N}\right)$. Since in general this bijection

$$
V_{N}: \mathscr{H}_{h}^{\mathscr{B}} \rightarrow \mathbf{H}_{N}
$$


is not unitary 8] we introduce $U_{N}=\left(V_{N} V_{N}^{*}\right)^{-\frac{1}{2}} V_{N}: \mathscr{H}_{h}^{\mathscr{B}} \rightarrow \mathbf{H}_{N}$. The sequence of spaces $\left(\mathbf{H}_{N}\right)_{N \in \mathbb{N}}$ must be viewed as the quantising Hilbert space for the reduced phase space $M$, and $U_{N}$ as a kind of Fourier integral operator allowing to transport equivariant wave functions of the original Bargmann space onto the reduced Hilbert space $\mathbf{H}_{N}$.

\subsection{Reduction of the Birkhoff normal form}

Let $K=K(\hbar)$ be an $\hbar$-pseudo-differential operator on $\mathbb{R}^{n}$ commuting with $\hat{H}_{2}$. Assume $K$ belongs to the class $\mathscr{M}_{\ell, m}$, as in definition 4.1, and consider again the family of rescaled operators $K(\epsilon, h): \mathscr{H}_{h} \rightarrow \mathscr{H}_{h}$. Since the Taylor expansion of the Weyl symbol $[K]$ is $\mathscr{O}_{\ell}$ we see as in (24) that the family $\epsilon^{-\ell} K(\epsilon, h)$ is continuous at $\epsilon=0$, if we set

$$
\epsilon^{-\ell} K(\epsilon, h)_{\lceil\epsilon=0}=K_{\ell}(h)
$$

where $K_{\ell}(h)$ is the differential operator with polynomial coefficients obtained by the leading term of $[K]$, as precisely defined in (24).

We wish to consider $K(\epsilon, h)$ as a reduced operator on $M$. For this purpose, let $K^{\mathscr{B}}(\epsilon, h)=U_{\mathscr{B}} K(\epsilon, h) U_{\mathscr{B}}^{*}$ and introduce

$$
T(\epsilon, h):=\epsilon^{-\ell} U_{N} K^{\mathscr{B}}(\epsilon, h) U_{N}^{*}: \mathbf{H}_{N} \rightarrow \mathbf{H}_{N} .
$$

Let $\boldsymbol{\Pi}_{N}$ denote the orthogonal projector on $\mathbf{H}_{N}$.

The main result of this section is that $T$ is a semiclassical Toeplitz operator on $M$, as stated in the following theorem.

Theorem 5.1 If $K \in \mathscr{M}_{\ell, m}$, then there exists a sequence of functions $(f(\cdot ; N))_{N \in \mathbb{N}}$ in $C^{\infty}\left(M \times\left[0, \epsilon_{0}\right]\right)$ admitting an asymptotic expansion

$$
f(x, \epsilon ; N)=f_{0}(x, \epsilon)+N^{-1} f_{1}(x, \epsilon)+N^{-2} f_{2}(x, \epsilon)+\cdots
$$

for the $C^{\infty}$ topology, such that

$$
T(\epsilon, h)=N^{-m} \Pi_{N} f(\cdot, \epsilon ; N)+\mathscr{O}\left(N^{-\infty}\right),
$$

uniformly in $\epsilon$. Moreover, if $k_{m}$ is the $\hbar$-principal symbol of $K$ then

$$
\epsilon^{\ell} f_{0}(\pi(z), \epsilon)=k_{m}(\epsilon z), \quad \forall z \in Y .
$$

Proof. The proof proceeds by first representing $K$ as a Toeplitz operator on $\mathbb{C}^{n}$ using the Bargmann transform and the corresponding formula for the Toeplitz symbol. Then one has to show that this symbol can be restricted to the energy hypersurface $H_{2}=1$ while retaining the asymptotics in $h$ (or $N^{-1}$ ) and the $S^{1}$ invariance. 
However, some technical preliminaries are required before this. As in the proof of lemma 4.2 we can assume that the symbol $k=k(x, \xi ; \hbar)$ is compactly supported. Again we introduce the rescaled symbol

$$
q(x, \xi ; \epsilon, h)=k\left(\epsilon x, \epsilon \xi ; \epsilon^{2} h\right) .
$$

Refining the proof of lemma 4.2 we can check that $q(\cdot, \epsilon, h)$ can be assumed to have a support $D$ that does not depend on $\epsilon$ and $h$ and with $0 \notin D$. Indeed, let $\Phi \in C_{0}^{\infty}\left(\mathbb{R}^{2 n}\right)$ with support not containing the origin and identically equal to 1 on a neighbourhood of $\left\{H_{2}=1\right\}$. By $h$-symbolic calculus

$$
\left(1-O p_{h}^{w}(\Phi)\right) \varphi\left(O p_{h}^{w}\left(H_{2}\right)\right)=\mathscr{O}\left(h^{\infty}\right),
$$

provided the support of $\varphi$ is suitably restricted around 1. Hence if we replace $q$ by the Weyl symbol of $O p_{h}^{w}(q) O p_{h}^{w}(\Phi)$, we deduce from equations (21) and (22) that $K(\epsilon, h)$ is modified by a term of order $\mathscr{O}\left(\epsilon^{\ell} h^{\infty}\right)$, which proves our claim. Moreover one has

$$
q(x, \xi ; \epsilon, h)=h^{m} \epsilon^{\ell} \tilde{q}(x, \xi ; \epsilon, h)
$$

where $\tilde{q}(\cdot, h)$ is a family of $C_{0}^{\infty}\left(\mathbb{R}^{2 n} \times\left[0, \epsilon_{0}\right]\right)$ admitting an asymptotic expansion in powers of $h$ for the $C^{\infty}$ topology. Hence, for the proof of the theorem, we can safely assume $m=\ell=0$.

We can now turn to the Bargmann side. Let

$$
Q^{\mathscr{B}}=U_{\mathscr{B}} O p_{h}^{w}(q(\cdot ; \epsilon, h)) U_{\mathscr{B}}^{*} .
$$

We know that $Q^{\mathscr{B}}$ can be represented as a Toeplitz operator. Precisely, let $\Pi^{\mathscr{B}}$ be the orthogonal projector of $L^{2}\left(\mathbb{C}^{n}, e^{-|z|^{2} / h}\right)$ onto $\mathscr{B}_{h}$. For any bounded function $g$ on $\mathbb{C}^{n}$, the Toeplitz operator with contravariant symbol $g$ is by definition the operator

$$
T_{g}: \mathscr{B}_{h} \rightarrow \mathscr{B}_{h}, \quad \psi \mapsto \Pi^{\mathscr{B}}(g \psi) .
$$

Then we have the following result

Theorem 5.2 If $g$ is in the symbol class $S(0)$ on $\mathbb{C}^{n} \simeq \mathbb{R}^{2 n}$ then the operator $U_{\mathscr{B}}^{*} T_{g} U_{\mathscr{B}}: L^{2}\left(\mathbb{R}^{n}\right) \rightarrow L^{2}\left(\mathbb{R}^{n}\right)$ is a pseudo-differential operator whose Weyl symbol is

$$
I(g)(\zeta)=\frac{1}{(\pi h)^{n}} \int_{\mathbb{C}^{n}} e^{-2 h^{-1}|z|^{2}} g(\zeta+z)|d z d \bar{z}| .
$$

The map I is continuous from $S(0)$ to $S(0)$. Moreover for any $g \in S(0)$ and all $k \geqslant 1$,

$$
I(g)=\sum_{j=0}^{k-1}\left(\frac{h}{2}\right)^{j} \Delta^{j} g / j !+h^{k} R_{k}(g)
$$

where $R_{k}$ is a continuous map from $S(0)$ to $S(0)$. 
Proof . Assume first that the symbol $g$ is in the Schwartz class. Since $U_{\mathscr{B}}^{*} U_{\mathscr{B}}=$ Id and $U_{\mathscr{B}} U_{\mathscr{B}}^{*}=\Pi^{\mathscr{B}}$, one has $U_{\mathscr{B}}^{*} T_{g} U_{\mathscr{B}}=U_{\mathscr{B}}^{*} g U_{\mathscr{B}}$ and its kernel is

$$
K(x, y)=\int_{\mathbb{C}^{n}} U_{\mathscr{B}}^{*}(x, z) g(z) U_{\mathscr{B}}(z, y) \mu(z)
$$

The kernel $U_{\mathscr{B}}(z, y)$ is given in $(\overline{28}), U_{\mathscr{B}}^{*}(x, z)=e^{-h^{-1}|z|^{2}} \overline{U_{\mathscr{B}}(z, x)}$ so

$$
K(x, y)=\frac{2^{n / 2}}{(2 \pi h)^{3 n / 2}} \int_{\mathbb{R}^{2 n}} e^{i h^{-1}\left(p \cdot(x-y)+\frac{i}{4}(x-y)^{2}+i\left(q-\frac{1}{2}(x+y)\right)^{2}\right)} g(q, p)|d q d p|
$$

with $z=(q-i p) / \sqrt{2}$. We recover the Weyl symbol $I(g)$ with the well-known formula

$$
\begin{aligned}
I(g)(x, \xi) & =\int_{\mathbb{R}^{n}} e^{-i h^{-1} v \cdot \xi} K\left(x+\frac{v}{2}, x-\frac{v}{2}\right)|d v| \\
& =\frac{2^{n / 2}}{(2 \pi h)^{3 n / 2}} \int_{\mathbb{R}^{3 n}} e^{i h^{-1}\left(v \cdot(p-\xi)+i(q-x)^{2}+\frac{i}{4} v^{2}\right)} g(q, p)|d q d p d v| \\
& =\frac{2^{n / 2}}{(2 \pi h)^{3 n / 2}} \int_{\mathbb{R}^{3 n}} e^{i h^{-1}\left(v \cdot p+\frac{i}{4} v^{2}+i q^{2}\right)} g(x+p, \xi+q)|d q d p d v| \\
& =\frac{1}{(\pi h)^{n}} \int_{\mathbb{R}^{2 n}} e^{-h^{-1}\left(p^{2}+q^{2}\right)} g(x+p, \xi+q)|d q d p|
\end{aligned}
$$

Going back to the coordinates $z$ and $\zeta=(x-i \xi) / \sqrt{2}$, we obtain the desired formula for $I(g)$. Next following the stationary phase method, we prove that the map $I$ is continuous $S(0) \rightarrow S(0)$ with the asymptotic expansion (32). Using a density argument, we conclude that the Weyl symbol of $U_{\mathscr{B}}^{*} T_{g} U_{\mathscr{B}}$ is $I(g)$ for any $g$ in the class $S(0)$.

Since the series involved in (32) is that of the exponential, it is easy to inverse formally. Hence let $g(\cdot, h)$ be a family in $C_{0}^{\infty}\left(\mathbb{C}^{n} \times\left[0, \epsilon_{0}\right]\right)$ with support in $D$ that admits the following asymptotic expansion:

$$
g(\cdot, h)=\sum\left(\frac{-h}{2}\right)^{j} \Delta^{j} q / j !+\mathscr{O}\left(h^{\infty}\right) .
$$

Then the proposition says that

$$
Q^{\mathscr{B}}=T_{g(\cdot ; \epsilon, h)}+\mathscr{O}\left(h^{\infty}\right)
$$

where the remainder is in the uniform norm. Notice that, since $h^{-1}$ is an affine function of $N$ with positive slope, asymptotics in $h$ are equivalent to asymptotics in $N^{-1}$. In particular we can neglect the remainder $\mathscr{O}\left(h^{\infty}\right)$. Therefore, restricting to $\mathscr{H}_{h}^{\mathscr{B}}$ we can express $K^{\mathscr{B}}(\epsilon, h)=Q_{\mathscr{H}_{h}^{\mathscr{B}}}^{\mathscr{B}}$ as

$$
K^{\mathscr{B}}(\epsilon, h): \mathscr{H}_{h}^{\mathscr{B}} \rightarrow \mathscr{H}_{h}^{\mathscr{B}}, \quad \psi \mapsto \boldsymbol{\Pi}^{\mathscr{H}_{h}^{\mathscr{B}}} g(\cdot ; \epsilon, h) \psi,
$$

where $\Pi^{\mathscr{H}_{h}^{\mathscr{B}}}$ is the orthogonal projector of $L^{2}\left(\mathbb{C}^{n}, e^{-|z|^{2} / h}\right)$ onto $\mathscr{H}_{h}^{\mathscr{B}}$. 
It remains to switch to the space $\mathbf{H}_{N}$ and see how to reduce the symbol $g=g(\cdot ; \epsilon, h)$ to the orbifold $M$.

The first step is to prove the theorem with $\left(V_{N}^{*}\right)^{-1}$ instead of $U_{N}$. In other words we look for a suitable symbol $J(g) \in C^{\infty}(M)$ such that

$$
\left(V_{N}^{*}\right)^{-1} K^{\mathscr{B}}(\epsilon, h) V_{N}^{-1}=\boldsymbol{\Pi}_{N} J(g),
$$

or, equivalently, inserting (34),

$$
\boldsymbol{\Pi}^{\mathscr{H}_{h}^{\mathscr{B}}} g=V_{N}^{*} \boldsymbol{\Pi}_{N} J(g) V_{N},
$$

acting on $\mathscr{H}_{h}^{\mathscr{B}}$. Using that $\boldsymbol{\Pi}^{\mathscr{H}_{h}^{\mathscr{B}}}$ and $\boldsymbol{\Pi}_{N}$ are self-adjoint, this amounts to show that for any $\psi, \psi^{\prime} \in \mathscr{H}_{h}^{\mathscr{B}}$

$$
\left\langle g \psi, \psi^{\prime}\right\rangle_{\mathbb{C}^{n}}=\left\langle J(g) V_{N} \psi, V_{N} \psi^{\prime}\right\rangle_{M}
$$

By definition

$$
\left\langle g \psi, \psi^{\prime}\right\rangle_{\mathbb{C}^{n}}=\int_{\mathbb{C}^{n}} e^{-|z|^{2} / h} g(z) \psi(z) \overline{\psi^{\prime}(z)} \mu(z),
$$

where $\mu$ is Lebesgue's measure on $\mathbb{C}^{n}=\mathbb{R}^{2 n}$. In order to decompose this integral, we use a slicing of $\mathbb{C}^{n}$ transversal to $Y$ given by the flow of the harmonic oscillator $H_{2}$ at imaginary times. Precisely, the map

$$
\mathbb{R} \times Y \rightarrow \mathbb{C}^{n} \backslash\{0\}, \quad(t, y) \mapsto z=\left(e^{t} \cdot y\right):=\left(e^{t \mathfrak{p}_{1}} y_{1}, \ldots, e^{t \mathfrak{p}_{n}} y_{n}\right)
$$

is a diffeomorphism. Now recall that $\mathscr{H}_{h}^{\mathscr{B}}$ is spanned by $z^{\alpha},\langle\alpha, \mathfrak{p}\rangle=N$. So any element $\Psi \in \mathscr{H}_{h}^{\mathscr{B}}$ satisfies

$$
\psi(u . z)=u^{N} \psi(z), \quad \forall u \in \mathbb{C} .
$$

Hence when $u=e^{t}$ one can write

$$
\left|\psi\left(e^{t} . z\right)\right|^{2} e^{-h^{-1}\left|e^{t} \cdot z\right|^{2}}=|\psi(z)|^{2} e^{-\nu_{c} N|z|^{2}} e^{-N \varphi(t, z)} e^{-\frac{|\nu|}{2}\left|e^{t} \cdot z\right|^{2}}
$$

with

$$
\varphi(t, z)=-2 t+\nu_{c} \sum\left(e^{2 \mathfrak{p}_{i} t}-1\right)\left|z_{i}\right|^{2} .
$$

Since the origin is not in the support of $g$ we shall now use the coordinates $(t, y) \in \mathbb{R} \times Y$ in order to calculate the integral (36). The measure $\mu$ can be decomposed as

$$
\mu=\delta(t, y)|d t| \mu_{Y}(y)
$$

where $\delta(t, y)$ is smooth and $\mu_{Y}$ is the Liouville measure of $Y$. Recall that $\mu_{Y}$ is $S^{1}$-invariant and $\pi_{*} \mu_{Y}=\mu_{M}$. Since $\mu$ is also $S^{1}$-invariant, the function 
$\delta(t, y)$ must be $S^{1}$-invariant as well. Finally remark that $g$ also, as defined in (33), inherits the $S^{1}$-invariance of $q$. This entails, together with (37), that

$$
\left\langle g \psi, \psi^{\prime}\right\rangle_{\mathbb{C}^{n}}=\int_{Y} e^{-\nu_{c} N|y|^{2}} J^{Y}(g)(y) \psi(y) \overline{\psi^{\prime}(y)} \mu_{Y}(y)
$$

where the function $J^{Y}(g) \in C^{\infty}(Y)$ is defined by

$$
J^{Y}(g)(y)=\int_{\mathbb{R}} e^{-N \varphi(t, y)} g(t, y) e^{-\frac{\nu}{2}|t y|^{2}} \delta(t, y)|d t| .
$$

Since $\varphi$ is $S^{1}$ invariant, so is $J^{Y}(g)$. Therefore there exists $J(g) \in C^{\infty}(M)$ such that $\pi^{*} J(g)=J^{Y}(g)$. Recall the definition (30) of the scalar product of $\mathbf{H}_{N}$. Since $\pi_{*} \mu_{Y}=\mu_{M}$, we get the desired identity (35).

Asymptotics of $J^{Y}(g)$ (and hence of $J(g)$ ) are obtained by the stationary phase lemma. Since $\varphi(t, y)$ has a global non-degenerate minimum at $t=0$, the expansion is localised on $Y$, as expected. For instance, at first order, we see that if $f_{0} \in C^{\infty}(M)$ is such that $\pi^{*} f_{0}(y)=g(0, y ; \epsilon, h)+\mathscr{O}(h)$ then

$$
J(g)(m)=e(m) f_{0}(m)+\mathscr{O}(h)
$$

where $e \in C^{\infty}(M)$ is a positive function.

To complete the proof of the theorem it remains to replace $\left(V_{N}^{*}\right)^{-1}$ by $U_{N}$. But since $U_{N}=\left(V_{N} V_{N}^{*}\right)^{-\frac{1}{2}} V_{N}$ we have

$$
U_{N} K^{\mathscr{B}}(\epsilon, h) U_{N}^{*}=\left(V_{N} V_{N}^{*}\right)^{\frac{1}{2}}\left(V_{N}^{*}\right)^{-1} K^{\mathscr{B}}(\epsilon, h) V_{N}^{-1}\left(V_{N} V_{N}^{*}\right)^{\frac{1}{2}}
$$

which means

$$
U_{N} K^{\mathscr{B}}(\epsilon, h) U_{N}^{*}=\left(V_{N} V_{N}^{*}\right)^{\frac{1}{2}}\left(\mathbf{\Pi}_{N} J(g)\right)\left(V_{N} V_{N}^{*}\right)^{\frac{1}{2}} .
$$

If we repeat our argument with $K(\epsilon, h)=\operatorname{Id}+\mathscr{O}\left(h^{\infty}\right)$ we see that

$$
\left(V_{N}^{*}\right)^{-1}\left(\operatorname{Id}+\mathscr{O}\left(h^{\infty}\right)\right) V_{N}^{-1}
$$

is a Toeplitz operator, and its principal symbol is $e$. We cannot omit here the remainder $\mathscr{O}\left(h^{\infty}\right)$ because we work with a compactly supported symbol for $K$. However, one can prove that the uniform norms of $N^{-\frac{1}{4}} V_{N}$ and its inverse are $\mathscr{O}(1)$ (cf. [B], proposition 4.22) Hence $\left(V_{N}^{*}\right)^{-1}\left(\mathscr{O}\left(h^{\infty}\right)\right) V_{N}^{-1}=\mathscr{O}\left(h^{\infty}\right)$ and thus is a Toeplitz operator (with asymptotically trivial symbol). Hence $W_{N}:=\left(V_{N}^{*}\right)^{-1} V_{N}^{-1}$ is a Toeplitz operator. By the symbolic and functional calculus of Toeplitz operator we get that

$$
U_{N} K^{\mathscr{B}}(\epsilon, h) U_{N}^{*}=W_{N}^{-\frac{1}{2}}\left(\boldsymbol{\Pi}_{N} J(g)\right) W_{N}^{-\frac{1}{2}}
$$

is indeed a Toeplitz operator with principal symbol $e^{-\frac{1}{2}}\left(e f_{0}\right) e^{-\frac{1}{2}}=f_{0}$. 


\subsection{Spectral asymptotics of eigenvalue clusters}

In this section we apply the previous result to the Birkhoff normal form of a pseudo-differential operator $P$, in order to get spectral asymptotics that we express in the original parameters $(E, \hbar)$. So we assume that $P$ satisfies the assumptions of theorem 3.11 with

$$
H_{2}(x, \xi)=\frac{\nu_{c}}{2} \sum_{j=1}^{n} \mathfrak{p}_{j}\left(x_{j}^{2}+\xi_{j}^{2}\right) .
$$

For the sake of simplicity, we shall also assume that $H_{0}=0$ : the subprincipal symbol of $P$ vanishes at $z_{0}$. Then, according to this theorem, the small eigenvalues of $P$ correspond to eigenvalues of $\hat{H}_{2}+K$, where $K$ commutes with $\hat{H}_{2}$.

Let $r$ be equal to 3 when there is a resonance relation of the form $\mathfrak{p}_{j}=2 \mathfrak{p}_{i}$ or $\mathfrak{p}_{i}=\mathfrak{p}_{j}+\mathfrak{p}_{k}$, and to 4 otherwise. Then $K$ belongs to $\mathscr{M}_{r, 0}$ and by lemma 4.2, the norm of the restriction of $K$ to the eigenspace $\mathscr{H}_{E, \hbar}$ of $\hat{H}_{2}$ is $\mathscr{O}\left(E^{\frac{r}{2}}\right)$.

Since the distance between two consecutive eigenvalues of $\hat{H}_{2}$ is larger than $\hbar \nu_{c}$, we conclude that the bottom of the spectrum of $P$ split into bands or clusters in this precise sense: there exists $\hbar_{0}>0$ and $C>0$ such that for every $\hbar \in\left(0, \hbar_{0}\right]$

$$
\operatorname{Sp}(P) \cap\left(-\infty, C \hbar^{\frac{2}{r}}\right) \subset \bigcup_{E \in \operatorname{Sp}\left(\hat{H}_{2}\right)}\left[E-\frac{\nu_{c} \hbar}{3}, E+\frac{\nu_{c} \hbar}{3}\right]
$$

Furthermore for any eigenvalue $E$ for $\hat{H}_{2}$ smaller than $C \hbar^{\frac{2}{r}}$,

$$
\# \mathrm{Sp}(P) \cap\left[E-\frac{\nu_{c} \hbar}{3}, E+\frac{\nu_{c} \hbar}{3}\right]=m(E, \hbar)
$$

with the multiplicity $m(E, \hbar)=\operatorname{dim} \mathscr{H}_{E, \hbar}$. First result is an estimate of the width of the band and of the distribution of the eigenvalues in each band in the regime $\hbar / E \rightarrow 0$. Denote by

$$
E+\lambda_{1}(E, \hbar) \leqslant \ldots \leqslant E+\lambda_{m(E, \hbar)}(E, \hbar)
$$

the eigenvalues of $P$ in $\left[E-\frac{\nu_{c} \hbar}{3}, E+\frac{\nu_{c} \hbar}{3}\right]$.

Let $k_{0}$ be the principal $\hbar$-symbol of $K$.

Theorem 5.3 For any $\hbar \in\left(0, \hbar_{0}\right]$ and any eigenvalue $E \leqslant C \hbar^{\frac{2}{r}}$ for $\hat{H}_{2}$, we have

$$
\begin{gathered}
\lambda_{1}(E, \hbar)=\inf _{x \in\left\{H_{2}=E\right\}}\left|k_{0}(x)\right|+E^{\frac{r}{2}} \mathscr{O}(\hbar / E), \\
\lambda_{m(E, \hbar)}(E, \hbar)=\sup _{x \in\left\{H_{2}=E\right\}}\left|k_{0}(x)\right|+E^{\frac{r}{2}} \mathscr{O}(\hbar / E)
\end{gathered}
$$


and for any function $g \in C^{\infty}(\mathbb{R})$,

$$
\sum_{i=1}^{m(E, \hbar)} g\left(\lambda_{i}(E, \hbar) / E^{\frac{r}{2}}\right)=\left(\frac{1}{2 \pi \hbar}\right)^{n-1} \int_{\left\{H_{2}=E\right\}} g\left(\frac{k_{0}(x)}{E^{\frac{r}{2}}}\right) \mu_{E}(x)+\mathscr{O}\left((E / \hbar)^{n-2}\right)
$$

where $\mu_{E}$ is the Liouville measure of $\left\{H_{2}=E\right\}$ and the $\mathscr{O}$ 's are uniform with respect to $\hbar$ and $E$.

To read this result, it is interesting to understand the dependence of the leading order terms with respect to $\hbar$ and $E$ as well. Recall that by remark 2.2 the Taylor expansion of $k_{0}$ is precisely the classical Birkhoff normal form of the principal symbol of $P$. We have $k_{0}=k^{(r)}+\mathscr{O}\left(|x, \xi|^{r+1}\right)$ with $k^{(r)}(x, \xi)$ a homogeneous polynomial of degree $r$. Then

$$
\begin{gathered}
\inf _{\left\{H_{2}=E\right\}}\left|k_{0}\right|=E^{\frac{r}{2}} \inf _{\left\{H_{2}=1\right\}}\left|k^{(r)}\right|+\mathscr{O}\left(E^{\frac{r+1}{2}}\right), \\
\int_{\left\{H_{2}=E\right\}} g\left(\frac{k_{0}(x)}{E^{\frac{r}{2}}}\right) \mu_{E}(x)=E^{n-1} \int_{\left\{H_{2}=1\right\}} g\left(k^{(r)}(x)\right) \mu_{1}(x)+\mathscr{O}\left(E^{n-\frac{1}{2}}\right) .
\end{gathered}
$$

If $r=3, k^{(r)}$ is easily computed. Let the Taylor expansion of the principal symbol of $P$ begin with $H_{2}+p^{(3)}+\mathscr{O}\left(|x, \xi|^{4}\right)$. Then $k^{(3)}$ is the average of $p^{(3)}$ with respect to the Hamiltonian flow of $H_{2}$. If $r=4$ then in general the formula is more involved : letting $p=H_{2}+p^{(3)}+p^{(4)}+\mathscr{O}\left(|x, \xi|^{5}\right)$ we see from the Birkhoff construction that $k^{(4)}$ is the average of $p^{(4)}-\frac{1}{2}\left\{p^{(3)}, a^{(3)}\right\}$, where $a^{(3)}$ is the Hamiltonian whose flow performs the first averaging in the method, ie. $\left\{H_{2}, a^{(3)}\right\}=-p^{(3)}$. We do not know a simpler formulation for this term, except, of course, when $p^{(3)}=0$.

Proof of the theorem. First the result holds for the eigenvalues

$$
\lambda_{1}^{\prime}(E, \hbar) \leqslant \ldots \leqslant \lambda_{m(E, \hbar)}^{\prime}(E, \hbar)
$$

of the restriction of $K$ to the eigenspace $\mathscr{H}_{E, \hbar}$. Indeed, by theorem 5.1, the numbers $\epsilon^{-r} \lambda_{i}^{\prime}\left(\epsilon^{2}, \epsilon^{2} h\right)$ are the eigenvalues of a Toeplitz operator which depends smoothly on $\epsilon$ and with semiclassical parameter $h$. The principal symbol of this operator is the push-forward to $M$ of the restriction of $\epsilon^{-r} k_{0}(\epsilon$.) to $\left\{H_{2}=1\right\}$ or equivalently of the restriction of $\epsilon^{-r} k_{0}($.$) to \left\{H_{2}=\epsilon^{2}\right\}$. The semiclassical estimates of the smallest and largest eigenvalue and of the spectral density of a Toeplitz operator in terms of its principal symbol are basic results which were extended to the orbifold case in [8]. Going back to the original parameter $E$ and $\hbar$, we obtain the result for the $\lambda_{i}^{\prime}(E, \hbar)$ and the $\mathscr{O}$ are uniform when $E$ and $\hbar$ run over an arbitrary bounded set.

Then when $E \leqslant C \hbar^{\frac{2}{r}}$ and $\hbar \in\left(0, \hbar_{0}\right]$ we have for any $N$

$$
\left|\lambda_{i}(E, \hbar)-\lambda_{i}^{\prime}(E, \hbar)\right| \leqslant C_{N}\left(E^{N}+\hbar^{N}\right)
$$


and we conclude easily. Since $\frac{1}{2} \nu_{c} \hbar \leqslant E \leqslant C \hbar^{\frac{2}{r}}$ the remainders $\mathscr{O}\left(E^{N}\right)$ and $\mathscr{O}\left(\hbar^{N}\right)$ are negligible when $N$ is sufficiently large and disappear.

Remark 5.4 In some sense the result is still true for higher energy $E$. When the condition $E \leqslant C \hbar^{\frac{2}{r}}$ is violated, the bands may overlap, and we can not extract the eigenvalues $\lambda_{i}(E, \hbar)$ from the whole spectrum of $P$ as we did in (38). However we saw in the proof that the eigenvalues $\lambda_{i}^{\prime}(E, \hbar)$ satisfy the same estimates as the $\lambda_{i}(E, \hbar)$ except that the remainders are uniform with respect to bounded energy $E$. Therefore the theorem remains valid in this regime provided $\lambda_{i}(E, \hbar)$ 's are replaced by $\lambda_{i}^{\prime}(E, \hbar)$ 's. Then we can recover the spectrum of $P$ with theorem 3.11 .

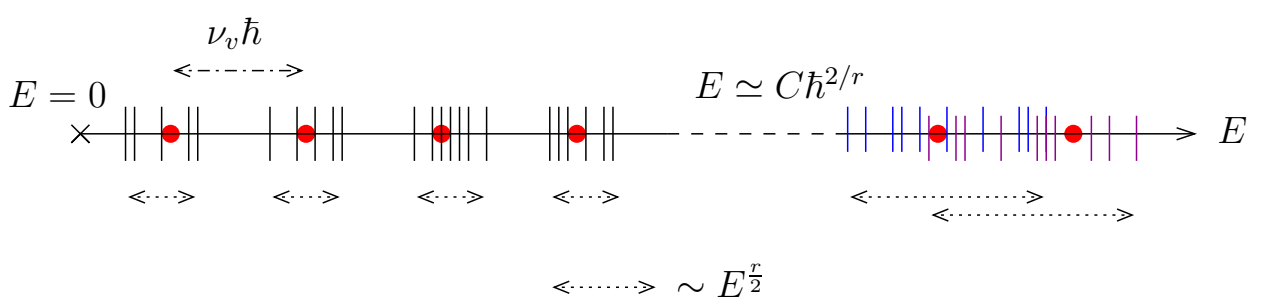

Figure 2: The spectrum (vertical ticks) exhibits clustering around the eigenvalues of the harmonic oscillator (big dots) when $E \leqslant C \hbar^{2 / r}$. But even when overlapping occurs, one can give a sense to each band if one thinks in terms of the eigenvalues $\lambda_{i}^{\prime}(E, \hbar)$. See remark 5.4 .

The estimate of the spectral density in the previous theorem is actually the leading order term of a full asymptotic expansion. The description of its various pieces is involved because the reduced phase space $M$ is not a manifold but an orbifold. First denote by $G$ the set of complex number $\zeta$ of modulus 1 whose fixed point set

$$
Y_{\zeta}:=\{z \in Y ; \zeta . z=z\}
$$

is not empty. Here the dot is for the Hamiltonian action generated by $\mathrm{H}_{2}$ as in (29). A straightforward computation leads to

$$
G=\left\{\zeta \in \mathbb{C}^{*} ; \zeta^{\mathfrak{p}_{i}}=1 \text { for some } i\right\}
$$

and

$$
Y_{\zeta}=\mathbb{C}_{\zeta} \cap Y \text { with } \mathbb{C}_{\zeta}=\left\{z \in \mathbb{C}^{n} ; z_{i}=0 \text { if } \zeta^{\mathfrak{p}_{i}} \neq 1\right\} .
$$

The $S^{1}$-action preserves $Y_{\zeta}$. Let $M_{\zeta}$ be the quotient $Y_{\zeta} / S^{1}$. It is a twisted projective space which embeds into $M$ as a symplectic suborbifold. Denote by $n(\zeta)$ its complex dimension. Finally, let $m(\zeta)$ be the greatest common divisor of $\left\{\mathfrak{p}_{i} ; \zeta^{\mathfrak{p}_{i}}=1\right\}$. Then with the same argument as in the previous proof we deduce from theorem 2.3 of [8] the 
Theorem 5.5 For every function $g \in C^{\infty}(\mathbb{R})$,

$$
\sum_{i=1}^{m(E, \hbar)} g\left(\lambda_{i}(E, \hbar) / E^{\frac{r}{2}}\right)=\sum_{\zeta \in G}\left(\frac{E}{2 \pi \hbar}\right)^{n(\zeta)} \zeta^{-N} \sum_{\ell=0}^{\infty}\left(\frac{\hbar}{E}\right)^{\ell} I_{\ell}\left(E^{\frac{1}{2}}, \zeta\right)+\mathscr{O}\left(\left(\frac{\hbar}{E}\right)^{\infty}\right)
$$

where the remainder is uniform when $\hbar$ and $E$ runs over $\left(0, \hbar_{0}\right)$ and $\operatorname{Sp}\left(\hat{H}_{2}\right) \cap$ $\left(-\infty, C \hbar^{\frac{2}{r}}\right)$ respectively, and $N$ is defined by $E=\hbar\left(\frac{\nu}{2}+N\right)$. Furthermore the coefficients $I_{\ell}(\epsilon, \zeta)$ are $C^{\infty}$ function of $\epsilon$ and

$$
I_{0}(\epsilon, \zeta)=\frac{1}{m(\zeta)}\left(\prod_{i ; \zeta^{\mathfrak{p}_{i}} \neq 1}\left(1-\zeta^{\mathfrak{p}_{i}}\right)^{-1}\right) \int_{M_{\zeta}} g\left(\epsilon^{-r} k_{0}(\epsilon x)\right) \mu_{\zeta}(x),
$$

where $\mu_{\zeta}$ is the Liouville measure of $M_{\zeta}$.

\subsection{A convex polytope and a trace formula}

As a consequence of the preceding theorems 5.3 and 5.5 we obtain an interesting formula expressing the asymptotics of a combinatoric sum over integral points of a convex polytope, when the polytope undergoes some rational scaling.

We first state the result without any reference to any operator, recalling only the following notation:

Let $n$ be a positive integer and let $\mathfrak{p}_{1}, \ldots, \mathfrak{p}_{n}$ be coprime positive integers. Let $\mathfrak{p}=\left(\mathfrak{p}_{1}, \ldots, \mathfrak{p}_{n}\right)$. Again let

$$
G=\left\{\zeta \in \mathbb{C}^{*} ; \zeta^{\mathfrak{p}_{i}}=1 \text { for some } i\right\}
$$

and for each $\zeta \in G$ introduce the index set $\mathbf{i}_{\zeta}=\left\{i ; \zeta^{\mathfrak{p}_{i}}=1\right\}$, whose cardinality is $n(\zeta)+1$. Finally recall that $m(\zeta)=\operatorname{gcd}\left\{\mathfrak{p}_{i} ; i \in \mathbf{i}_{\zeta}\right\}$.

For $N \in \mathbb{N}$ and $\alpha \in \mathbb{N}^{n}$, we define the convex polytope (actually a simplex of dimension $n-1$ )

$$
\mathcal{P}(\alpha, N)=\left(\mathbb{R}^{+}\right)^{n} \cap\left\{\left(x_{1}, \ldots, x_{n}\right) ; \quad\langle x, \mathfrak{p}\rangle=N-\langle\alpha, \mathfrak{p}\rangle\right\} .
$$

Remark 5.6 Notice that $\mathcal{P}(\alpha, N)$ is neither integral or Delzant (in the terminology of [15]). However if $p$ is the least common multiple of $\mathfrak{p}_{1}, \ldots, \mathfrak{p}_{n}$ then $\mathcal{P}(p \alpha, p N)$ has vertices with integral coordinates, but it still not Delzant in general. This reflects the fact that $\mathcal{P}(\alpha, N)$ is a moment polytope for a symplectic orbifold which - except for $\mathfrak{p}_{1}=\cdots=\mathfrak{p}_{n}=1$ - is not a manifold.

When $\alpha=\left(\alpha_{1}, \ldots, \alpha_{n}\right) \in \mathbb{N}^{n}$ we use the notation $\alpha !=\left(\alpha_{1}\right) ! \cdots\left(\alpha_{n}\right) !$. 
Theorem 5.7 For any $\alpha \in \mathbb{N}^{n}$ we have the asymptotic formula

$$
\frac{1}{N^{|\alpha|}} \sum_{\gamma \in \mathcal{P}(\alpha, N) \cap \mathbb{Z}^{n}} \frac{(\gamma+\alpha) !}{\gamma !}=\sum_{\zeta \in G} \zeta^{-N} N^{n(\zeta)} \sum_{\ell=0}^{\infty} N^{-\ell} a_{\ell}(\alpha, \zeta)+\mathscr{O}\left(N^{-\infty}\right) .
$$

Moreover

$$
a_{0}(\alpha, \zeta)=\frac{\prod_{i \notin \mathbf{i}_{\zeta}}\left(1-\zeta^{p_{i}}\right)^{-1}}{m(\zeta) \prod_{i \in \mathbf{i}_{\zeta}} \mathfrak{p}_{i}^{\alpha_{i}+1}} \frac{\prod_{i \in \mathbf{i}_{\zeta}} \Gamma\left(\alpha_{i}+1\right)}{\Gamma\left(\sum_{i \in \mathbf{i}_{\zeta}}\left(\alpha_{i}+1\right)\right)} .
$$

Remark 5.8 As we shall see below, the $a_{0}(\alpha, \zeta)$ 's are actually "Weyl terms", in the sense that they are the result of some integrals over different faces of the polytope (or, equivalently, they are phase space integrals for some sub-orbifolds).

Remark 5.9 If we choose $\alpha=0$, the left-hand-side of is just the number of integral points of the polytope $\mathcal{P}(0, N)$, and the formula becomes:

$$
\#\left(\mathcal{P}(0, N) \cap \mathbb{Z}^{n}\right)=\sum_{\zeta \in G} \zeta^{-N} N^{n(\zeta)} \sum_{\ell=0}^{\infty} N^{-\ell} b_{\ell}(\zeta)+\mathscr{O}\left(N^{-\infty}\right)
$$

where

$$
b_{0}(\zeta)=a_{0}(0, \zeta)=\frac{\prod_{i \notin \mathbf{i}_{\zeta}}\left(1-\zeta^{p_{i}}\right)^{-1}}{m(\zeta)\left(\prod_{i \in \mathbf{i}_{\zeta}} \mathfrak{p}_{i}\right) \Gamma(n(\zeta)+1)}
$$

It is known that each factor of $\zeta^{-N}$ is actually a polynomial in $N$ given by a Riemann-Roch type formula. See [21] for details on this issue. The leading term, obtained with $\zeta=1$ is

$$
\frac{N^{n-1}}{\left(\mathfrak{p}_{1} \cdots \mathfrak{p}_{n}\right)(n-1) !}
$$

The other coefficients can also be obtained as the coefficients of the generating function $g(X)=\prod_{i=1}^{n}\left(1-X^{\mathfrak{p}_{i}}\right)^{-1}$; see [25].

Proof of the theorem. Let us work in the Bargmann representation. The harmonic oscillator we consider is

$$
\hat{H}_{2}(\hbar)=\hbar \sum_{j=1}^{n}\left(\mathfrak{p}_{j} \frac{\partial}{\partial z_{j}}+\frac{1}{2}\right) .
$$


If $\alpha$ and $\beta$ are multi-indices in $\mathbb{N}^{n}$, the differential operator $z^{\alpha}\left(\hbar \frac{\partial}{\partial z}\right)^{\beta}$ commutes with $\hat{H}_{2}(\hbar)$ if and only if $\langle\alpha-\beta, \mathfrak{p}\rangle=0$. Thus let us consider the symmetric differential operators

$$
K_{\alpha}(\hbar):=z^{\alpha}\left(\hbar \frac{\partial}{\partial z}\right)^{\alpha}
$$

We shall compute the trace of the restriction of $K_{\alpha}$ to the eigenspace $\mathscr{H}_{E, \hbar}$ in two different ways. The first way is just to do an explicit computation in a basis of $\mathscr{H}_{E, \hbar}$. The second way is to use remark 5.4 in order apply theorem 5.5 with $g=\mathrm{Id}$ and $E=1$.

1. - A basis of $\mathscr{H}_{E, \hbar}$ (or, more exactly, of the space $\mathscr{H}_{E, \hbar}^{\mathscr{B}}$ in the Bargmann representation) is given by the monomials

$$
\left\{z^{\gamma} ; \quad \gamma \geqslant 0 \text { and }\langle\gamma, \mathfrak{p}\rangle=N\right\}
$$

where the integer $N$ is defined by the equation $E=\hbar(N+|\mathfrak{p}| / 2)$, and the inequality $\gamma \geqslant 0$ stands for $\gamma_{j} \geqslant 0, \forall j$. It is straightforward to check that

$$
K_{\alpha}(\hbar)\left(z^{\gamma}\right)= \begin{cases}\hbar^{|\alpha|} \frac{\gamma !}{(\gamma-\alpha) !} z^{\gamma} & \text { if } \gamma \geqslant \alpha \\ 0 & \text { otherwise. }\end{cases}
$$

Hence

$$
\operatorname{Tr}\left(K_{\alpha}(\hbar)_{\mid \mathscr{H}_{E, \hbar}}\right)=\hbar^{|\alpha|} \sum_{\substack{\gamma \geqslant \alpha \\\langle\gamma, \boldsymbol{p}\rangle=N}} \frac{\gamma !}{(\gamma-\alpha) !}=\hbar^{|\alpha|} \sum_{\gamma \in \mathcal{P}(\alpha, N) \cap \mathbb{Z}^{n}} \frac{(\gamma+\alpha) !}{\gamma !} .
$$

2. - Formula (40) is now a simple transcription of theorem 5.5, using $E=1$ and $\hbar=1 /(N+|\mathfrak{p}| / 2)$, and rewriting the coefficients in order to transform the expansion in powers of $\left(\frac{\hbar}{E}\right)$ into an expansion in powers of $N^{-1}$. This of course does not change the formulas for the leading coefficients.

Let us compute these leading coefficients. For this we use some homogeneity property in the variable $E$, so it is best not to set $E=1$ for the moment.

We introduce the standard action-angle coordinates for the harmonic oscillator in $\mathbb{R}^{2 n}$. Let $I_{j}=\frac{1}{2}\left(x_{j}^{2}+\xi_{i}^{2}\right)=\left|z_{j}\right|^{2}$, and let the angles $\theta_{j}$ be defined by

$$
\left\{\begin{array}{l}
x_{j}=\sqrt{2 I_{j}} \cos \theta_{j} \\
\xi_{j}=-\sqrt{2 I_{j}} \sin \theta_{j} .
\end{array}\right.
$$

Since $d \xi_{j} \wedge d x_{j}=d I_{j} \wedge d \theta_{j}$ and the singular set of these action-angle coordinates is of codimension 2 , we can use the corresponding symplectic measure on $\left(\mathbb{R}^{+}\right)^{n} \times(\mathbb{R} / 2 \pi \mathbb{Z})^{n}:\left|d I_{1} \wedge \cdots \wedge d I_{n} \wedge d \theta_{1} \wedge \cdots \wedge d \theta_{n}\right|$ as a replacement for the symplectic measure $\mu$ on $\mathbb{R}^{2 n}$. Let $|d E|$ denote the pull-back by 
$H_{2}=\sum_{j} \mathfrak{p}_{j} I_{j}$ of the Lebesgue measure on $\mathbb{R}$. Since the flow of $H_{2}$ is $2 \pi$ periodic, the Liouville measure $\mu_{E}$ on $\left\{H_{2}=E\right\}$ is by definition the quotient of $\mu$ by $2 \pi|d E|$ :

$$
\mu=2 \pi \mu_{E} \otimes|d E| .
$$

The principal symbol of $K_{\alpha}(\hbar)$ is $k_{0, \alpha}=z^{\alpha} \bar{z}^{\alpha}=\prod I_{j}^{\alpha_{j}}$, for which we use the notation $I^{\alpha}$. Let $S_{\alpha}(E)=\int_{\left\{H_{2}=E\right\}} I^{\alpha} \mu_{E}$.

Since $d E \wedge d I_{2} \wedge \cdots \wedge d I_{n}=\mathfrak{p}_{1} d I_{1} \wedge \cdots \wedge d I_{n}$ we see that

$$
\mu_{E}=\left(2 \pi \mathfrak{p}_{1}\right)^{-1}\left|d I_{2} \wedge \cdots \wedge d I_{n} \wedge d \theta_{1} \wedge \cdots \wedge d \theta_{n}\right|
$$

and

$$
S_{\alpha}(E)=(2 \pi)^{n-1} \mathfrak{p}_{1}^{-1} \int_{\mathfrak{p}_{1} I_{1}+\cdots \mathfrak{p}_{n} I_{n}=E} I^{\alpha}\left|d I_{2} \cdots d I_{n}\right|=E^{|\alpha|+n-1} S_{\alpha}(1) .
$$

We conclude by adapting the standard trick used to calculate the surface of the unit sphere, namely let

$$
J=\int_{0}^{\infty} e^{-E} S_{\alpha}(E)|d E| .
$$

On the one hand,

$$
J=S_{\alpha}(1) \int_{0}^{\infty} e^{-E} E^{|\alpha|+n-1}|d E|=S_{\alpha}(1) \Gamma(|\alpha|+n) ;
$$

on the other hand

$$
\begin{aligned}
J & =\int_{\left(\mathbb{R}^{+}\right)^{n}} e^{-H_{2}(I)} I_{1}^{\alpha_{1}} \cdots I_{N}^{\alpha_{n}}\left|d I_{1} \cdots d I_{n}\right| \\
& =\left(\int_{0}^{\infty} e^{-\mathfrak{p}_{1} I_{1}}\left|d I_{1}\right|\right) \cdots\left(\int_{0}^{\infty} e^{-\mathfrak{p}_{n} I_{n}}\left|d I_{n}\right|\right) .
\end{aligned}
$$

Hence

$$
S_{\alpha}(1)=\frac{\Gamma\left(\alpha_{1}+1\right) \cdots \Gamma\left(\alpha_{n}+1\right)}{\mathfrak{p}_{1}^{\alpha_{1}+1} \cdots \mathfrak{p}_{n}^{\alpha_{n}+1} \Gamma(|\alpha|+n)} .
$$

This gives the result for $a_{0}(\alpha, 1)$. For a general $\zeta$ the calculation of the integral over $M_{\zeta}$ is exactly the same as the integral $S_{\alpha}(1)$ provided we keep only indices $i \in \mathbf{i}_{\zeta}$, as follows from the definition of $Y_{\zeta}$ in (39). This finishes the proof.

Remark 5.10 Instead of $K_{\alpha}(\hbar)$, we could have considered the more general operators commuting with $\hat{H}_{2}(\hbar): z^{\alpha}\left(\hbar \frac{\partial}{\partial z}\right)^{\beta}$, with $\langle\alpha-\beta, \mathfrak{p}\rangle=0$. But using the basis $z^{\gamma}$ as before it is easy to see that the trace of such operators always vanishes as soon as $\alpha \neq \beta$. As a consequence, this shows that for any $\zeta \in G$,

$$
\int_{M_{\zeta}} z^{\alpha} \bar{z}^{\beta} \mu_{\zeta}=0 \quad \text { provided }\langle\alpha-\beta, \mathfrak{p}\rangle=0, \quad \alpha \neq \beta .
$$




\section{References}

[1] M. K. Ali. The quantum normal form and its equivalents. J. Math. Phys., 26(10):2565-2572, 1985.

[2] D. Bambusi. Semiclassical normal forms. In Multiscale methods in quantum mechanics, Trends Math., pages 23-39. Birkhäuser Boston, Boston, MA, 2004.

[3] D. Bambusi, S. Graffi, and T. Paul. Normal forms and quantization formulae. Commun. Math. Phys., 207(1):173-195, 1999.

[4] V. Bargmann. On a Hilbert space of analytic functions and an associated integral transform I. Comm. Pure Appl. Math., 19:187-214, 1961.

[5] G.D. Birkhoff. Dynamical systems. AMS, 1966.

[6] L. Boutet de Monvel and V. Guillemin. The spectral theory of Toeplitz operators. Number 99 in Annals of Mathematics Studies. Princeton university press, 1981.

[7] L. Charles. Berezin-toeplitz operators, a semi-classical approach. Commun. Math. Phys., 239(1-2):1-28, 2003.

[8] L. Charles. Toeplitz operators and Hamiltonian torus actions. Jour. Funct. Analysis, 2006. To appear.

[9] Y. Colin de Verdière. Méthodes semi-classiques et théorie spectrale. (in preparation), 2006.

[10] Y. Colin de Verdière and S. Vũ Ngọc. Singular Bohr-Sommerfeld rules for 2D integrable systems. Ann. Sci. École Norm. Sup. (4), 36:1-55, 2003.

[11] M. Dimassi and J. Sjöstrand. Spectral asymptotics in the semi-classical limit, volume 268 of London Mathematical Society Lecture Note Series. Cambridge University Press, Cambridge, 1999.

[12] H. Dulac. Solutions d'un système d'équations différentielles dans le voisinage de valeurs singulières. Bull. Soc. Math. France, 40:324-383, 1912 .

[13] B. Eckhardt. Birkhoff-Gustavson normal form in classical and quantum mechanics. J. Phys. A, 19:2961-2972, 1986.

[14] B. Fedosov. Deformation quantization and index theory, volume 9 of Mathematical Topics. Akademie Verlag, Berlin, 1996. 
[15] V. Guillemin. Moment maps and combinatorial invariants of Hamiltonian $T^{n}$-spaces, volume 122 of Progress in Mathematics. Birkhäuser Boston Inc., Boston, MA, 1994.

[16] V. Guillemin. Wave-trace invariants. Duke Math. J., 83(2):287-352, 1996.

[17] F. G. Gustavson. On constructing formal integrals of a Hamiltonian system near an equilibrium point. Astron. J., 71:670-686, 1966.

[18] B. Helffer and D. Robert. Calcul fonctionnel par la transformation de Mellin et opérateurs admissibles. J. Funct. Anal., 53(3):246-268, 1983.

[19] B. Helffer and J. Sjöstrand. Multiple wells in the semi-classical limit. I. Comm. Partial Differential Equations, 9:337-408, 1984.

[20] M. Joyeux. Gustavson's procedure and the dynamics of highly excited vibrational states. J. Chem. Phys., 109:2111-2122, 1998.

[21] E. Meinrenken. On Riemann-Roch formulas for multiplicities. J. Amer. Math. Soc., 9(2):373-389, 1996.

[22] J. Moser. New aspects in the theory of Hamiltonian systems. Comm. Pure Appl. Math., 9:81-114, 1958.

[23] D. Robert. Autour de l'approximation semi-classique, volume 68 of Progress in Mathematics. Birkhäuser, 1987.

[24] J. Robert and M. Joyeux. Canonical perturbation theory versus BornOppenheimer-type separation of motions : the vibrational dynamics of C3. J. Chem. Phys., 119:8761-8762, 2003.

[25] D.A. Sadovskií and B. Zhilinskií. Counting levels within vibrational polyads. J. Chem. Phys., 103(24), 1995.

[26] B. Simon. Semiclassical analysis of low lying eigenvalues I. Ann. Inst. H. Poincaré. Phys. Théor., 38(3):295-307, 1983. a correction in 40:224.

[27] J. Sjöstrand. Semi-excited states in nondegenerate potential wells. Asymptotic Analysis, 6:29-43, 1992.

[28] R. T. Swimm and J. B. Delos. Semiclassical calculations of vibrational energy levels for nonseparable systems using the birkhoff-gustavson normal form. J. Chem. Phys., 71(4):1706-1717, 1979.

[29] S. Vũ Ngọc. Sur le spectre des systèmes complètement intégrables semiclassiques avec singularités. PhD thesis, Université Grenoble 1, 1998.

[30] S. Zelditch. Inverse Spectral Problem for Surfaces of Revolution. J. Differential Geom., 49:207-264, 1998. 